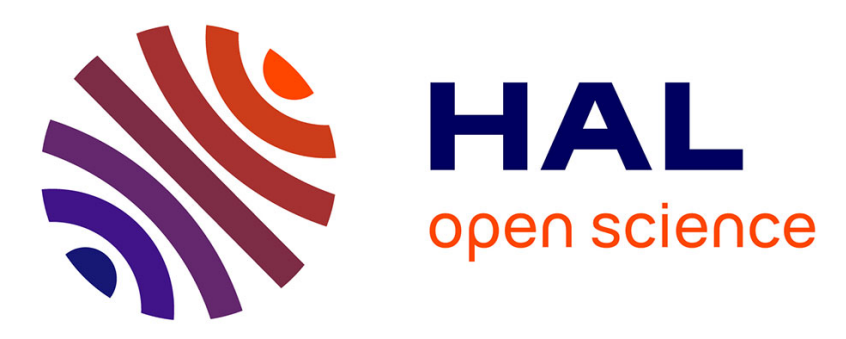

\title{
Heterogeneous perimeter flow distributions and MFD-based traffic simulation
}

Guilhem Mariotte, Ludovic Leclercq

\section{To cite this version:}

Guilhem Mariotte, Ludovic Leclercq. Heterogeneous perimeter flow distributions and MFD-based traffic simulation. Transportmetrica B: Transport Dynamics, 2019, 29p. 10.1080/21680566.2019.1627954 . hal-02160465

\section{HAL Id: hal-02160465 \\ https://hal.science/hal-02160465}

Submitted on 19 Jun 2019

HAL is a multi-disciplinary open access archive for the deposit and dissemination of scientific research documents, whether they are published or not. The documents may come from teaching and research institutions in France or abroad, or from public or private research centers.
L'archive ouverte pluridisciplinaire HAL, est destinée au dépôt et à la diffusion de documents scientifiques de niveau recherche, publiés ou non, émanant des établissements d'enseignement et de recherche français ou étrangers, des laboratoires publics ou privés. 


\title{
RESEARCH ARTICLE
}

\section{Heterogeneous perimeter flow distributions and MFD-based traffic simulation}

\author{
Guilhem Mariotte $^{\mathrm{a}}$ and Ludovic Leclercq ${ }^{\mathrm{a}}$ \\ aUniv. Lyon, ENTPE, IFSTTAR, LICIT, F-69518, Lyon, France
}

\section{ARTICLE HISTORY}

Compiled June 11, 2019

\begin{abstract}
This paper investigates how network and traffic heterogeneities influence the accuracy of a simulation based on the Macroscopic Fundamental Diagram (MFD). To this end, the MFD modeling of a simple grid network is compared to the outputs of a mesoscopic kinematic wave model simulating traffic in the same network. Heterogeneous distributions of demand and supply at the boundaries are set to the local entries and exits of the mesoscopic model to generate heterogeneous network loadings. These boundary conditions challenge the MFD simulation, as significant discrepancies are observed between both modeling approaches in steady state. While the accurate calibration of the MFD and the average trip length can reduce the discrepancies for heterogeneous demand settings, no simple solution exists for heterogeneous supply settings, because they may drive very different internal congestion patterns in the network. We propose a correction method to adjust the MFD model outputs in such a case.

(C) 2019. The authors. This manuscript version is made available under the CC-

BY-NC-ND 4.0 license

(c) BY-NC-ND http://creativecommons.org/licenses/by-nc-nd/4.0/

Accepted for publication in Transportmetrica B: Transport Dynamics

DOI: $10.1080 / 21680566.2019 .1627954$. Available online at www.tandfonline.com
\end{abstract}

\section{KEYWORDS}

Macroscopic Fundamental Diagram; Heterogeneous network loading; Reservoir perimeter; Average trip length; Urban network simulation

\section{Context and study framework}

\subsection{The impact of network heterogeneities on the MFD}

The Macroscopic Fundamental Diagram (MFD) has received much attention in the literature over the last decade, in particular for control applications, (e.g. KeyvanEkbatani et al. 2012; Aboudolas and Geroliminis 2013; Haddad and Mirkin 2017; Ampountolas, Zheng, and Geroliminis 2017). Partitioning a city into a multi-reservoir system characterized by MFDs also appears to be an appealing and computationally efficient method for simulating urban multimodal networks at a large scale (e.g. Knoop and Hoogendoorn 2014; Yildirimoglu, Ramezani, and Geroliminis 2015). The concept of MFD was first introduced by Godfrey (1969), Herman and Prigogine (1979) and Mahmassani, Williams, and Herman (1984). However, Daganzo and Geroliminis 
(2008) were the first authors to present a well-defined MFD based on real data. Since then, numerous studies have investigated the conditions for good MFD definition. It has notably been shown that network and/or traffic heterogeneities are strong sources of MFD scattering. Heterogeneities can be driven by: (i) non-consistent network definition (Buisson and Ladier 2009), (ii) data sources and processing methods (Leclercq, Chiabaut, and Trinquier 2014), (iii) the spatial distribution of vehicles within the network (Mazloumian, Geroliminis, and Helbing 2010; Geroliminis and Sun 2011b; Saberi and Mahmassani 2012), (iv) time of the day (Gayah and Daganzo 2011), (v) network layout and configuration (Knoop, De Jong, and Hoogendoorn 2014; Ortigosa, Menendez, and Gayah 2015; Muhlich, Gayah, and Menendez 2015), (vi) traffic signal settings (de Jong, Knoop, and Hoogendoorn 2013; Zhang, Garoni, and de Gier 2013; Gayah, Gao, and Nagle 2014; Girault et al. 2016), (vii) the definition of the Origin-Destination (OD) matrix (Doig, Gayah, and Cassidy 2013; Leclercq et al. 2015) and (viii) traveler information (Zhao et al. 2014). Furthermore, heterogeneities may be observed not only inside the area but also at its border. Keyvan-Ekbatani et al. (2016) notably focused on balancing queues at a network perimeter to improve its gating, and showed how this manipulation of boundary conditions may affect the MFD shape.

\subsection{How does this affect the accuracy of MFD-based simulation?}

In the above-mentioned studies, the main focus is often on the impact the MFD shape or existence. However, little attention has been paid to assess the effect of heterogeneities on the accuracy of MFD-based simulation. As heterogeneities not only affect the MFD shape but also the distance traveled in an area (Leclercq et al. 2015; Batista, Leclercq, and Geroliminis 2019), the impacts on simulation results may be even more considerable. A simple way to assess the accuracy of MFD-based simulation results is to compare them with those of more refined traffic flow models using microscopic (e.g. Gipps 1986; Barcelo et al. 2006) or mesoscopic approaches (e.g. Ben-Akiva et al. 2002; Mahut, Florian, and Tremblay 2003; Burghout, Koutsopoulos, and Andreasson 2005; Leclercq and Becarie 2012). Geroliminis and Daganzo (2007) were the first to compare MFD simulation in a single region (also called "reservoir") with a microscopic simulator on a 2.5 square mile area of Downtown San Francisco. They showed that the results from the reservoir model fit the microsimulation outputs quite well, mainly because the network studied exhibits a low-scattered MFD which seems independent from OD tables. But this low scattering is not universally expected, as suggested by the above-mentioned studies on network heterogeneities. Yildirimoglu and Geroliminis (2014) also compared the evolution of the perimeter flow estimated by a multi-reservoir MFD model with microscopic simulation. The results are more mitigated as consistency is obtained by integrating a feedback mechanism that adjusts the accumulation in the MFD model from time to time, depending on the current state predicted by the microscopic model. In this paper, the authors also clearly pinpointed that assuming a single and constant trip length for all vehicles within a network may lead to strong bias with the microscopic simulation results. This was recently corroborated by Paipuri, Leclercq, and Krug (2019). Such an assumption was first conjectured by Daganzo (2007). Since then, most papers that have used MFD simulation (mostly for control applications) have taken advantage of this simplification. This can be supported by the promising results of the perimeter control applied in microsimulation by KeyvanEkbatani, Papageorgiou, and Papamichail (2013); Keyvan-Ekbatani, Papageorgiou, and Knoop (2015) with a simple MFD model, which benefits from a good homogene- 
ity of traffic states in the network studied. However, recent results (e.g. Yildirimoglu and Geroliminis 2014; Leclercq et al. 2015; Lamotte and Geroliminis 2018) are now questioning the simple MFD model with a constant average trip length for the most general cases. They show that not only traffic conditions but also the OD matrix may significantly affect the mean travel distance in a network.

\subsection{Towards a better understanding of MFD-based simulation accuracy in an heterogeneously loaded network}

In this study we aim to characterize how MFD-based simulation is sensitive to network heterogeneities generated by uneven demand and supply settings at the local entries and exits that define the perimeter of a single reservoir (i.e. an urban region). In the following, for the sake of simplicity we use the terms "exit supply" or sometimes "supply" to mean the capacity of the exit nodes (expressed in [veh/s]). Such capacities can simulate downstream link-level restrictions due to spillbacks from an adjacent region for instance. The question of uneven perimeter loading is very critical for MFD simulation, because this modeling approach automatically considers homogeneous loading as all entry and exit link flows are aggregated. By definition of the single reservoir model first introduced in Daganzo (2007), only one inflow and one outflow are plugged into the MFD simulation. If heterogeneity at the perimeter can be catch in this framework, this can only be visible in the MFD and/or the average trip length estimation. Therefore, our study addresses the following steps: (i) first investigate the impact of heterogeneous distributions of demand and supply on a network MFD and average trip length, (ii) then analyze and quantify the resulting errors on traffic state predictions by MFD simulation, and (iii) finally propose some methods to correct the inaccuracies in MFD simulation. The main contributions are thus (i) a better understanding on the impact of uneven perimeter loading on network performances, and (ii) a step forward the validation of MFD-based simulation with clearer insights on some shortcomings that have been overlooked in the literature.

\subsection{General layout and methodology}

Our methodology is based on simulation comparison to assess the accuracy of MFD modeling under uneven loading. MFD simulation results for a single region are compared with a mesoscopic simulator (Leclercq and Becarie 2012) capable of reproducing local dynamic effects in the network and in particular spillbacks between intersections during congestion. This link-level simulator is used in our study as the ground truth to identify possible limitations in the MFD model. Our case study uses the same $2 \times 4$ network as presented in Leclercq et al. (2015) because this network, although very simplistic, permits generating a wide range of different heterogeneous loadings. Its features (asymmetrical shape, one-way streets, etc) are deliberately exaggerated to emphasize heterogeneities and thus identify possible shortcomings while simulating traffic states with the MFD. This network is not meant to be general, dense and homogeneous, but rather to represent a simplified version of the irregular and asymmetrical reservoir shapes often exhibited by partitioning algorithms (see e.g. Ji and Geroliminis 2012; Saeedmanesh and Geroliminis 2016). We focus on the ability of the MFD model to predict accurate traffic states in the steady phase, i.e. after the initial network loading. Comparisons are based on the dynamic evolution of the vehicle accumulation within the reservoir, and inflows and outflows. 
This paper is organized as follows. Section 2 introduces the case study and the two modeling approaches, the mesoscopic one in subsection 2.1, and the MFD-based one in subsection 2.2. In particular, we present the demand and supply settings and how we define homogeneous or heterogeneous distributions of flows at the network perimeter. In section 3 then, the simulation findings are presented in two parts: subsection 3.1 is about the MFD and average trip length estimations made with the link-level simulations for different boundary settings, and subsection 3.2 deals with the comparison of the simulation outputs in steady state between the two modeling scales. Guidelines will be proposed on the best way to set up the MFD-based model. Finally, Section 4 discusses the major findings of this study while presenting new results obtained on a bigger network.

\section{The network studied and its settings}

\subsection{Mesoscopic simulation settings}

\subsubsection{Network configuration and geometry}

In this study, we focus on a very simple $2 \times 4$ Manhattan-type network where all the links are one-way and have one lane. Thus, the network has 6 entries and 6 exits, see Figure 1(a). The West-East inner links are more than twice as long as the North-South ones. All the intersections are controlled by traffic lights with the same green and red times equal to $30 \mathrm{~s}$. All the signals have the same offset. This asymmetrical network configuration has been designed to favor heterogeneous loadings. The geometry ensures highly variable trip lengths as a function of the OD matrix, as the West-East routes are much longer than the North-South ones (Leclercq et al. 2015). Furthermore, uneven supply distributions at the exits will trigger many different congestion patterns. We keep the network configuration simple as a large number of mesoscopic simulations must be run in order to investigate the influence of the demand and supply distribution over the entries and exits.

\subsubsection{Origin-Destination matrix}

Since our aim is to investigate the impact of boundary loadings on MFD-based simulation, we consider only transferring trips for the sake of simplicity. By ignoring internal trips, we decided to limit the number of potential parameters in the demand settings to keep the analysis simple. Moreover, the treatment of internal trips might differ from that of transferring trips in MFD-based simulation. Consequently, considering only the latter allows omitting this research question which is out of the scope of this study. Thus, in the following, origins and destinations refer to the 6 entries and 6 exits defining the network perimeter, see also Figure 1(a). In the whole study, the distribution of destinations among users starting from a unique origin remains constant, i.e. the coefficients of the normalized OD matrix in Figure 1(b) are time-invariant. Such a distribution guarantees that homogeneous demand loadings (same demand at all entries) will correspond to a homogeneous flow distribution at the exits. This does not imply a homogeneous use of the network however. Here, the OD flow matrix depends only on the demand values assigned to each entry. 


\subsubsection{Traffic simulation settings}

The mesoscopic simulator in this study is fully described in Leclercq and Becarie (2012); Joueiai et al. (2015). This simulator is based on the space-Lagrangian resolution of the LWR model (Lighthill and Whitham 1955; Richards 1956) and provides the exact solutions of this model for links (Laval and Leclercq 2013). At the link level, traffic flow is characterized by a triangular fundamental diagram (FD) with the following parameters: free-flow speed $u=25 \mathrm{~m} / \mathrm{s}$, wave speed $w=5 \mathrm{~m} / \mathrm{s}$, and jam density $k_{j}=0.2 \mathrm{veh} / \mathrm{m}$. Outputs of one simulation provide the passing times of all the vehicles at each node.

\subsubsection{Assignment and convergence}

One simulation lasts $5000 \mathrm{~s}$ including a $1000 \mathrm{~s}$ warm-up period. For all local OD pairs, a maximum of the three shortest routes in distance may be used by vehicles. The flow distribution over the different routes is calculated by the mesoscopic simulator based on the first Wardrop principle (user equilibrium), i.e. all users travel on the paths with the minimum travel time. To distribute the users between the different routes, different simulations are run iteratively using the Method of Successive Averages (MSA). The travel time values are based on the free-flow speed for the first simulation, and on the previous simulation output for the next iterations. The traffic assignment process converges once the mean travel time of $95 \%$ of all the link lengths is almost constant (less than $5 \%$ variation over two successive iterations).

(a)

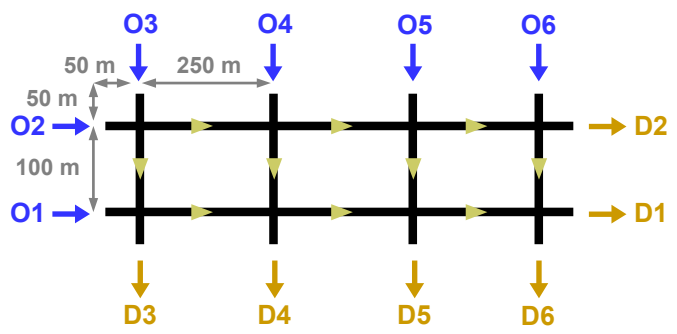

(b)

\begin{tabular}{c|cccccc|}
\multicolumn{1}{c}{} & D1 & D2 & D3 & D4 & D5 & D6 \\
\cline { 2 - 7 } O1 & $1 / 6$ & 0 & $1 / 3$ & $1 / 4$ & $1 / 4$ & 0 \\
O2 & 0 & $1 / 6$ & $1 / 3$ & $1 / 6$ & $1 / 6$ & $1 / 6$ \\
O3 & 0 & 0 & $1 / 3$ & $1 / 4$ & $1 / 6$ & $1 / 4$ \\
O4 & $1 / 4$ & $1 / 4$ & 0 & $1 / 3$ & $1 / 6$ & 0 \\
O5 & $1 / 4$ & $1 / 4$ & 0 & 0 & $1 / 4$ & $1 / 4$ \\
O6 & $1 / 3$ & $1 / 3$ & 0 & 0 & 0 & $1 / 3$ \\
\cline { 2 - 7 } & & & & & &
\end{tabular}

Figure 1. (a) Network configuration. (b) Normalized origin-destination matrix

\subsubsection{Demand scenarios}

A wide range of constant demand values at the entries and constant supply (capacity) values at the exits are applied to this network. The reference scenario, named SC0 hereon, corresponds to a uniform distribution between the entries for the total demand and uniform distribution between the exits for the total supply, see Figure 2(a). The demand at each entry is equal to $q_{\text {in }}^{D}$ (in [veh/s]) and the supply at each exit is $q_{\text {out }}^{S}$ (in $[\mathrm{veh} / \mathrm{s}]$ ). Heterogeneous network loadings will be obtained by either using uneven demand distribution over all the entries (scenario SC1), see Figure 2(b), or uneven supply distribution at the exits (scenario SC2), see Figure 2(c). Demand and supply patterns are considered linear by the network directions. For a given value of $q_{\mathrm{in}}^{D}$ (or $q_{\mathrm{out}}^{S}$ in the case of scenario SC2), a single parameter $a_{1}$ (or $a_{2}$ in the case of scenario $\mathrm{SC} 2)$ which ranges from 0 to 1 is thus sufficient to define the demand values at all the entries (or the supply values at all the exits for SC2), see Figure 2(b) and (c). 
Parameters $a_{1}$ and $a_{2}$ are called the heterogeneity coefficients. Note that $a_{1}$ and $a_{2}=$ 0 for SC0. Also note that the sum of the demand flow on all the entries (or the sum of the flow restrictions on all the exits) is always the same, i.e. independent from the heterogeneity coefficients. It is equal to $Q_{\mathrm{in}}^{D}=6 q_{\mathrm{in}}^{D}$ for the demand, and to $Q_{\mathrm{out}}^{S}=6 q_{\mathrm{out}}^{S}$ for the supply.

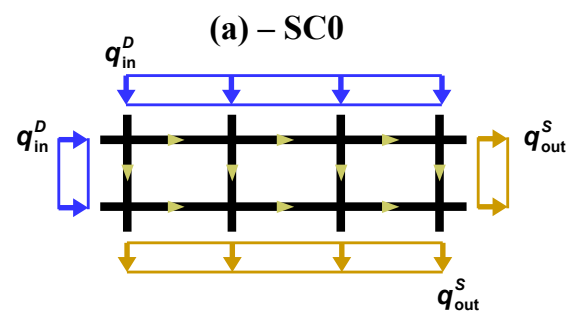

(b) - SC1

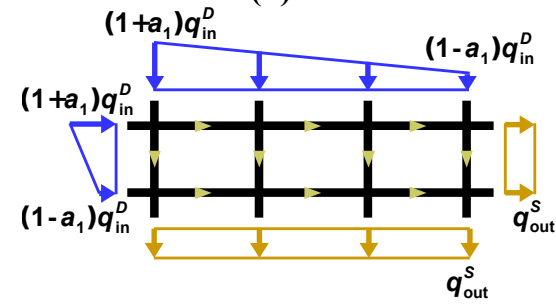

(c) $-\mathrm{SC2}$

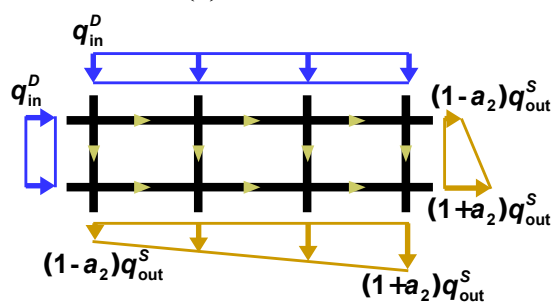

Figure 2. (a) Scenario SC0: Homogeneous demand and supply distribution. (b) Scenario SC1: Heterogeneous demand distribution at the entries with homogeneous supply distribution at the exits. (c) Scenario SC2: Heterogeneous supply distribution at the exits with homogeneous demand distribution at the entries.

\subsection{Single reservoir implementation of the MFD-based model}

In parallel to the mesoscopic simulations, the traffic states in the network are estimated using an MFD-based model (Daganzo 2007; Geroliminis and Daganzo 2007). Here, we consider that the whole network is described by a single reservoir with a total inflow $Q_{\text {in }}(t)$ and a total outflow $Q_{\text {out }}(t)$ (in [veh/s]). Traffic states within the reservoir are characterized by the vehicle accumulation $n(t)$ (in [veh]), which is the number of vehicles circulating at time $t$. Cumulative count curves $N_{\text {in }}(t)$ and $N_{\text {out }}(t)$ (in [veh]) are also calculated by respectively integrating inflows and outflows to monitor the reservoir perimeter. The network MFD is defined as the relationship between the travel production $P(n)$ (in [veh.m/s]) and the accumulation $n$. The existence of a well-defined MFD will be tested against the mesoscopic simulation results in the next section 3.1.1. According to Daganzo (2007), the evolution of $n(t)$ is given by the following conservation equation:

$$
\frac{d n}{d t}=Q_{\text {in }}(t)-Q_{\text {out }}(t)
$$

The same author makes the following crucial assumption: the outflow $Q_{\text {out }}(t)$ is proportional to the MFD travel production $P(n(t))$, where the proportionality factor is the inverse of the average trip length $L$ (in $[\mathrm{m}]$ ) in the reservoir, as detailed in equation 2. This means that all vehicles have almost the same travel distance whatever 
their origins and destinations.

$$
Q_{\text {out }}(t)=\frac{P(n(t))}{L}
$$

When capacity restrictions are applied to the exits, the outflow formulation must be updated to account for the exogenous boundary condition (total supply) $Q_{\text {out }}^{S}$; see equation 3. Similarly, the total demand inflow $Q_{\text {in }}^{D}$ may be reduced to adjust the reservoir supply function $I(n(t))$, see equation 4 . Note that $Q_{\text {in }}(t)$ is set at the maximal demand value during the whole period during which a stock of vehicles is waiting to enter the reservoir due to capacity supply regulation. Note also that we do not consider internal flow generation in the reservoir as this does not occur in the mescoscopic simulation.

$$
\begin{aligned}
Q_{\text {out }}(t) & =\min \left[\frac{P(n(t))}{L} ; Q_{\text {out }}^{S}\right] \\
Q_{\text {in }}(t) & =\min \left[I(n(t)) ; Q_{\mathrm{in}}^{D}\right]
\end{aligned}
$$

The definition of the supply or entrance function $I(n(t))$ is introduced in Geroliminis and Daganzo (2007) and its shape is presented in Figure 3(b). The role of this function is to simulate spillbacks reaching the reservoir entry when studying transfer flows through a reservoir. However, considerations of its use and shape are still being discussed in the literature ${ }^{1}$. Thus, as there is no clear consensus on this question and to keep our model simple, we chose to adopt the definition of Knoop and Hoogendoorn (2014); Hajiahmadi et al. (2013); Lentzakis, Ware, and Su (2016), which is based on the supply function of a cell in the Cell Transmission Model (CTM) of Daganzo (1994), see equation 5 . Since our goal is to study steady state flows in this paper, this choice does not have any impact on the final results. We will actually see that the role of $I(n(t))$ is basically to ensure that the equilibrium flow is equal to the total supply $Q_{\text {out }}^{S}$ in congestion for the MFD-based simulation.

$$
I(n)= \begin{cases}P_{c} / L=\max O(n) & \text { if } n<n_{c}=\operatorname{argmax} O(n) \\ O(n) & \text { else }\end{cases}
$$

where $O(n)=P(n) / L$ is the outflow function, and $P_{c}$ the maximum network production estimated with the MFD $P(n) . O(n)$ may be also referred to as the outflow-MFD, while $P(n)$ is denoted the production-MFD to avoid confusion. Such a distinction was proposed in Lamotte and Geroliminis (2018).

Figure 4 synthesizes the MFD-based modeling approach. For a given and constant set of boundary conditions $\left(Q_{\mathrm{in}}^{D} ; Q_{\mathrm{out}}^{S}\right)$, the network steady state can be directly calculated by solving $Q_{\text {in }}(t)=Q_{\text {out }}(t)$. Note that at this aggregated level, neither the total demand $Q_{\mathrm{in}}^{D}$ nor the total supply $Q_{\text {out }}^{S}$ depends on the heterogeneity coefficients $a_{1}$ and $a_{2}$. This is because the MFD-based model considers homogeneous perimeter flows. Thus in this model, the network steady state depends only on $\left(Q_{\mathrm{in}}^{D} ; Q_{\mathrm{out}}^{S}\right)$, the production-MFD $P(n)$ and the average trip length $L$. However, a crucial question is whether $P(n)$ or $L$ may be affected by heterogeneous network loadings and therefore by $a_{1}$ or $a_{2}$. This will be investigated in the next section.

\footnotetext{
${ }^{1}$ Most large-scale control applications do not even consider it because of the feedback loop introduced with the controller, thus protecting the reservoir from over-saturated states. But in our case, $I(n(t))$ is needed to
} 
(a)

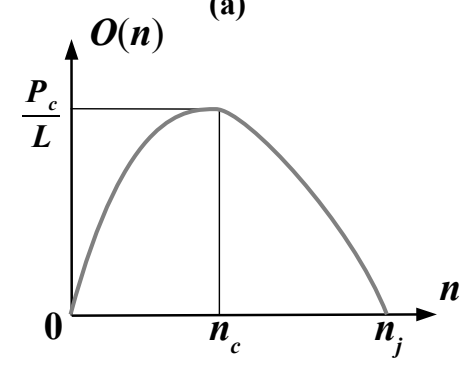

(b)

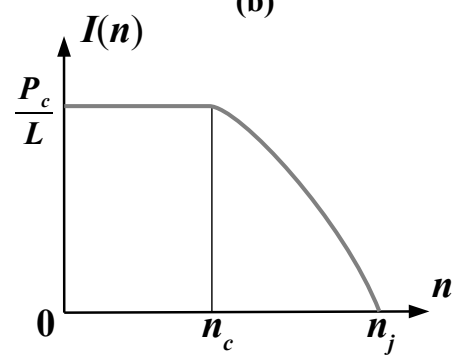

Figure 3. (a) Typical shape of the outflow function $O(n)$ and (b) the supply or entrance function $I(n)$

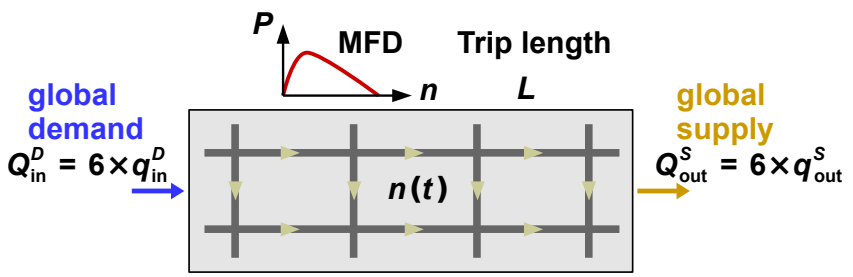

Figure 4. MFD-based model definition

\section{Simulation findings}

\subsection{Influence of the boundary settings on the MFD and the average trip length}

\subsubsection{MFD estimation based on the mesoscopic outputs}

The production-MFD $P(n)$ is estimated by simulating different traffic loadings with the mesoscopic framework. One simulation corresponds to one particular loading, and the steady state reached after the warm-up period defines a single point in the (accumulation, production) plane. The different states for the free-flow part of the MFD are obtained from simulations with local demand values varying from $q_{\mathrm{in}}^{D}=0.1$ to $0.5 \mathrm{veh} / \mathrm{s}$ and a supply value fixed to $q_{\mathrm{out}}^{S}=1 \mathrm{veh} / \mathrm{s}$ at each exit. On the other hand, the dots for the congested part of the MFD are obtained by varying the supply at the exits from $q_{\text {out }}^{S}=0.1$ to $1 \mathrm{veh} / \mathrm{s}$ and fixing the demand at its highest level $q_{\text {in }}^{D}=$ $0.5 \mathrm{veh} / \mathrm{s}$ at each entry. These two simulation settings allow us to almost completely estimate the network MFD.

For one simulation, the steady state accumulation is calculated as the mean difference between the entering and exiting cumulative count curves during the time period $[1000 ; 5000 \mathrm{~s}]$. The count curves are direct outputs from the simulation because the mesoscopic simulator provides all the entering and exiting times for all vehicles. On the other hand, the steady state production is calculated as the mean circulating flow $Q_{\text {circu }}$ multiplied by the total network length $L_{\text {net }}$. $Q_{\text {circu }}$ is obtained with Edie's formula (Edie 1963):

$$
Q_{\text {circu }}=\frac{1}{L_{\mathrm{net}} T_{\mathrm{obs}}} \sum_{i=1}^{N_{\mathrm{veh}}} d_{i}
$$


where $T_{\text {obs }}$ is the duration of the observation period $[1000 ; 5000 \mathrm{~s}], N_{\mathrm{veh}}$ the total number of vehicles circulating during the same period, and $d_{i}$ the distance traveled by the $i$ th vehicle during this period.

The MFD is estimated for homogeneous loadings (SC0) but also for different degrees of heterogeneous loadings (SC1 and SC2), i.e. when coefficient $a_{1}$ varies from 0 to 0.8 , and $a_{2}$ from 0 to 0.6. The resulting MFDs and their fits by a semi-parabola and semi-linear function can be seen in Figures 5(a) and (b). A clear impact of the heterogeneity coefficients appears on the MFD shape. Figure 5(a) shows the results for SC1. It can be seen that the more heterogeneous the demand on entries is, the lower the MFD capacity is. This is because the flows concentrate on a few entries when the demand distribution becomes heterogeneous. In this case certain OD pairs are almost not used. This has no effect when the demand rates are low, but creates uneven density distribution between the links when the demand reaches its highest level. This uneven density distribution is therefore responsible for the decrease in the networks global capacity, as shown previously by Geroliminis and Sun (2011a); Knoop, Van Lint, and Hoogendoorn (2015) in bigger networks. Zhang, Garoni, and de Gier (2013) also studied the influence of anisotropic demand settings at network boundaries on the MFD shape. Likewise, their simulations show a decrease in the network capacity when anisotropy exists in the demand distribution.

On the other hand, heterogeneity among the supply values at the exits does not affect the MFD capacity, see Figure 5(b). This is because the network capacity is reached for network loadings where the exit flows are not limited, whatever the heterogeneity coefficient $a_{2}$ is. However, a clear decrease of the average flow in the congested part is observed. This underlines that traffic states become even worse as congestion increases, when the heterogeneity coefficient $a_{1}$ is high. Reducing outflow at some exits may lead to severe congestion on several network links, which may also affect other exits when spillbacks occur.

Figures 5(c) and (d) show the increase of spatial heterogeneity among link traffic states when the spatial mean density increases for both scenarios SC1 and SC2. These two graphs are based on the same data points we used for the MFD estimations. For one simulation, link densities are calculated as the mean of link accumulation over the period $[1000 ; 5000 \mathrm{~s}]$, divided by the link length. Then, the spatial mean density $k$ and the spatial link heterogeneity $\sigma_{k}$ respectively correspond to the arithmetic mean and the standard deviation of all these link densities. This definition of spatial link heterogeneity has already been used in Zhang, Garoni, and de Gier (2013); Zhao et al. (2014); Knoop, Van Lint, and Hoogendoorn (2015). Figure 5(c) shows the results for $\mathrm{SC} 1$. The more heterogeneous the demand distribution is, the more heterogeneous the link traffic states. On the contrary, Figure 5(d) shows the results for SC2. It obviously appears that high heterogeneity coefficients for supply values lead to highly heterogeneous link densities in congestion. In free-flow however, the heterogeneity coefficient $a_{2}$ has no impact, see also Figure 5(b). In both figures, a linear trend can also be seen between the spatial mean density $k$ and the spatial heterogeneity $\sigma_{k}$. This trend was noticed by Knoop, Hoogendoorn, and Van Lint (2012), and by Mahmassani, Hou, and Saberi (2013); Kim and Mahmassani (2015) about the variability in travel times.

\subsubsection{Mean trip length estimation based on the mesoscopic outputs}

The average trip length $L$ can be estimated in two ways. First, the mesoscopic model provides the trip distances directly for all exiting vehicles. The average trip length for 

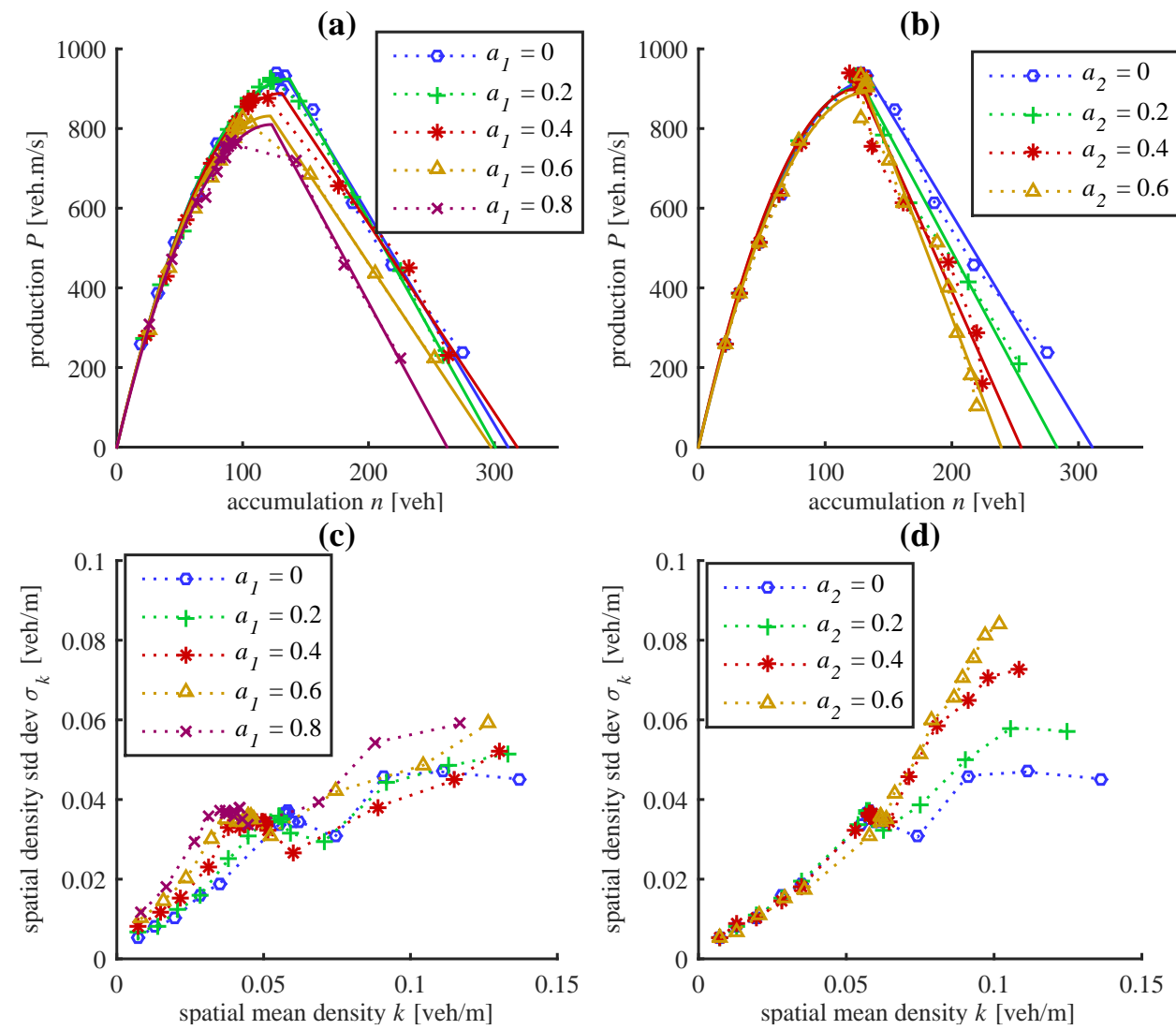

Figure 5. (a) MFD estimation for SC1 (heterogeneous demand / homogeneous supply), $a_{1}=0$ to 0.8 . (b) MFD estimation for SC2 (homogeneous demand / heterogeneous supply), $a_{2}=0$ to 0.6. (c) Spatial heterogeneity (standard deviation) of link densities vs spatial mean link density in SC1 with $a_{1}=0$ to 0.8 , and (d) in $\mathrm{SC} 2$ with $a_{2}=0$ to 0.6

a given simulation can then be calculated as the arithmetic mean of these distances during the observation period. This estimation is denoted $L_{\text {simul }}$ as it refers to one single simulation setting. The second method consists in determining the slope of the linear fit between the outflow and the production values; see equation 2. For each level of heterogeneity, i.e. a couple of $a_{1}$ and $a_{2}$ values, a linear regression is applied to all the simulation results to estimate the mean trip length, here referred to as $L_{\mathrm{MFD}}$ because it corresponds to one MFD estimation.

$L_{\text {simul }}$ values are presented in Figures 6(a) and (b) for the two scenarios SC1 and $\mathrm{SC} 2$ respectively. For each simulation, $L_{\text {simul }}$ is given versus the corresponding steady state accumulation. Figures 6(c) and (d) show the same data points, but versus the heterogeneity coefficient $a_{1}$ (resp. $a_{2}$ ). The network state, i.e. free-flow or congested, related to the MFD estimation (see section 3.1.1) is mentioned in the figure. The second estimation $L_{\mathrm{MFD}}$ is also presented in these two graphs for each heterogeneity coefficient, including the $95 \%$ confidence interval of the slope estimation in the linear regression. It clearly appears that the average trip length greatly depends on the boundary conditions and may have significant variability (from 350 to $500 \mathrm{~m}$ ). An interesting observation is that although the confidence intervals are small for the $L_{\mathrm{MFD}}$ values, the second estimation method proved to be insufficient to capture the great variability that may exist between the different simulations. Other network-specific trends of lesser importance can also be noticed. Such observations are more specific 
to the network studied and should not be expected as universal results. First, one counter-intuitive phenomenon is that the average trip length decreases as congestion becomes heavier, because the inflow is more reduced drastically for the longest routes when congestion propagates. Second, the heterogeneity in the demand distribution at entries has a clear impact on the average trip length in free-flow: Figures 6(a) and (c) show that higher heterogeneity coefficient values increase the user flow on longer routes, see also Figure 2(b). On the other hand, in Figures 6(b) and (d) the average trip length is independent of the heterogeneity coefficient in free-flow $\left(a_{2}\right.$ characterizes the heterogeneity on supply). This is because the demand is homogeneous in scenario SC2. The impact of these differences in MFD and/or trip length estimations on the reservoir simulation will be examined next.
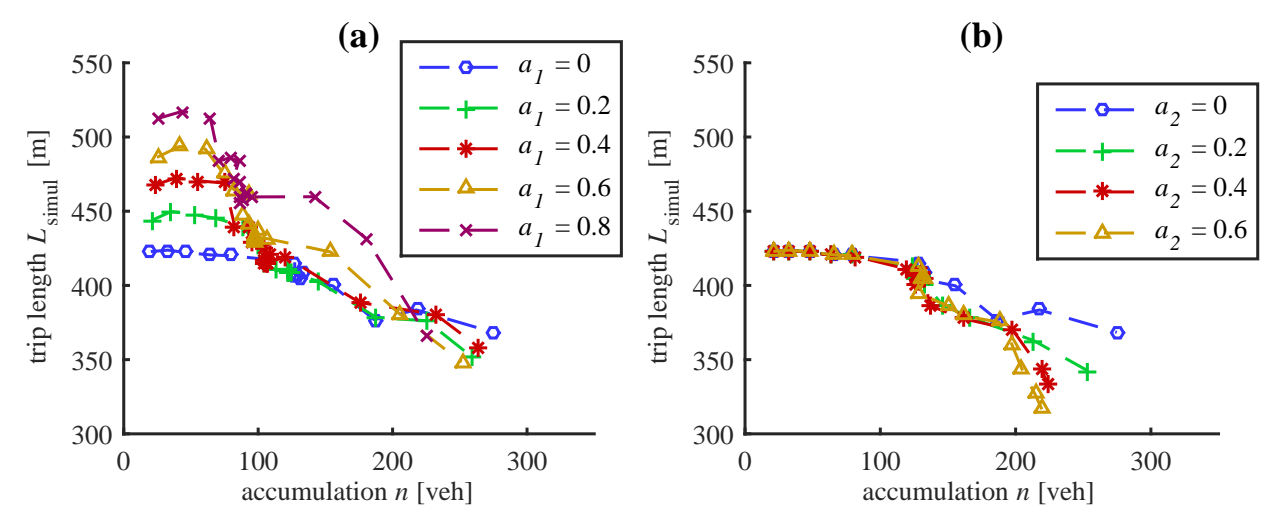

(c)

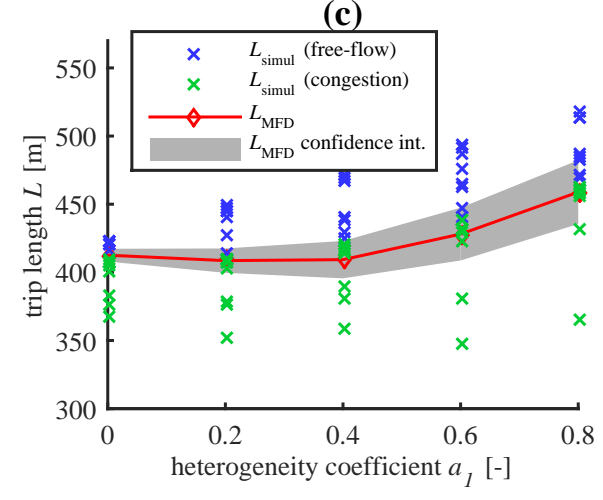

(d)

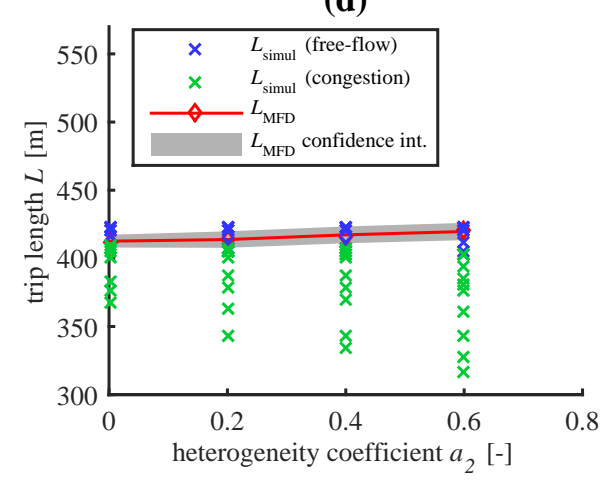

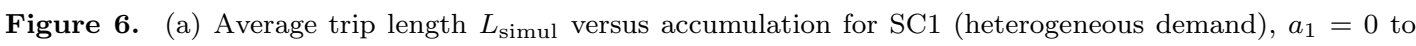

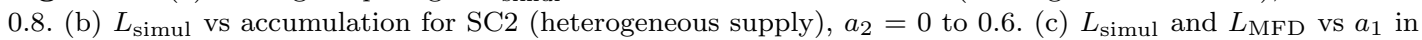
SC1. (d) $L_{\text {simul }}$ and $L_{\mathrm{MFD}}$ vs $a_{2}$ in SC2

\subsection{Comparisons of MFD and mesoscopic simulation results for different network loading cases}

\subsubsection{Comparison between mesoscopic and MFD-based approaches for homogeneous loadings}

In this section we present the simulation results on a simple network loading case: the simulation starts with an empty network and then reaches a steady state defined by the demand level in free-flow, or the supply level in congestion. Both levels are 
constant during the whole simulation period. We first focus on two typical network loadings: (i) the free-flow case, defined by $q_{\mathrm{in}}^{D}=0.2 \mathrm{veh} / \mathrm{s}$ and $q_{\mathrm{out}}^{S}=1 \mathrm{veh} / \mathrm{s}$; (ii) the congested case, characterized by $q_{\mathrm{in}}^{D}=0.5 \mathrm{veh} / \mathrm{s}$ and $q_{\mathrm{out}}^{S}=0.1 \mathrm{veh} / \mathrm{s}$. These are the reference values for one entry or one exit in SC0. The effective local inflows and outflows are then derived based on the $a_{1}$ and $a_{2}$ coefficients in SC1 and SC2, see section 2.1. At the aggregated (reservoir) level, we have $Q_{\mathrm{in}}^{D}=1.2 \mathrm{veh} / \mathrm{s}$ and $Q_{\mathrm{out}}^{S}=$ $6 \mathrm{veh} / \mathrm{s}$ for free-flow, and $Q_{\mathrm{in}}^{D}=3 \mathrm{veh} / \mathrm{s}$ and $Q_{\mathrm{out}}^{S}=0.6 \mathrm{veh} / \mathrm{s}$ for congestion.

We first compare the simulation results between the mesoscopic and MFD-based approaches for the homogeneous case $\mathrm{SC} 0$, when $a_{1}$ and $a_{2}$ are set to 0 . The evolution of accumulation and $N$-curves are presented in Figures 7(a) and (b) for the free-flow situation, and in Figures 7(c) and (d) for the congested situation. Overall, a good concordance is observed between the two modeling frameworks in steady state. Of course, the traffic description at the aggregated (reservoir) level cannot capture all the variability which is rendered at the lower (link) level by the mesoscopic simulator. Note that the scattering is considerable in congestion for the mesoscopic results.

(a)

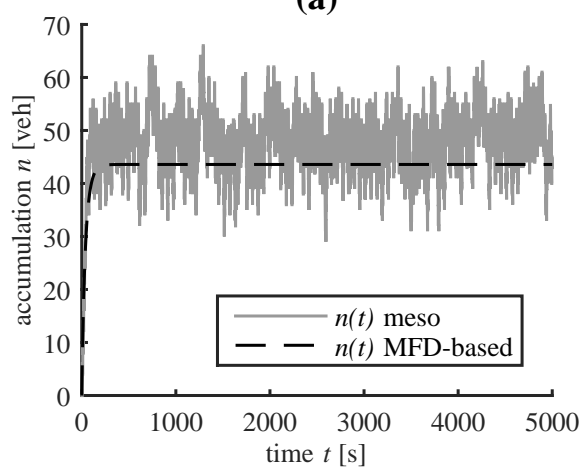

(c)

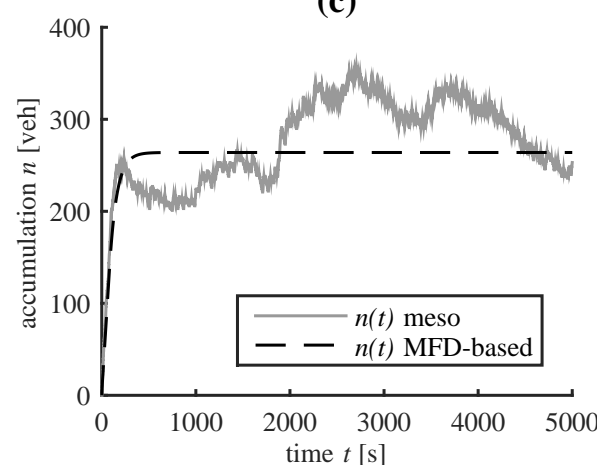

(b)

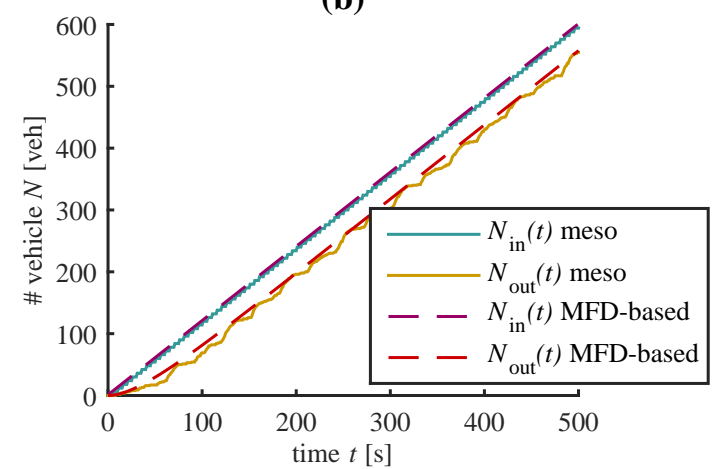

(d)

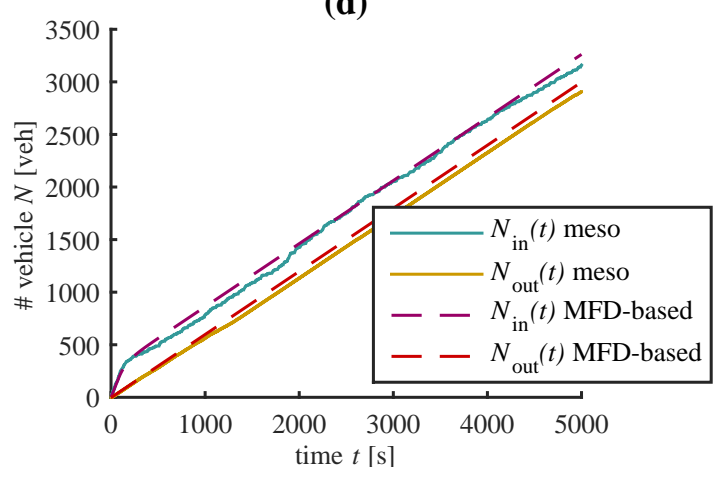

Figure 7. Comparison between the mesoscopic and the MFD-based framework when $a_{1}=0$ and $a_{2}=0$. (a) Evolution of accumulation $n(t)$ for the free-flow case (b) $N_{\text {in }}(t)$ and $N_{\text {out }}(t)$ for the free-flow case (the time window was reduced to improve the graph's readability). (c) Evolution of accumulation $n(t)$ for the congested case (d) $N_{\text {in }}(t)$ and $N_{\text {out }}(t)$ for the congested case

\subsubsection{Comparison between mesoscopic and MFD-based approaches for heterogeneous demand and homogeneous supply loadings - scenario SC1}

We now focus on the impact of heterogeneity in $\mathrm{SC} 1$, by setting the $a_{1}$ value to 0.8 (highly heterogeneous distribution) and $a_{2}$ to 0 for both free-flow and congested situations. Several options are available for calibrating the reservoir model, i.e. the 
MFD shape and the mean trip length value. We can either consider that the reservoir model has been calibrated for the homogeneous case, i.e. when $a_{1}=0$ and that we are not aware of the change of the demand distribution; or we can re-calibrate the reservoir model using heterogeneous data observations. We consider updating the MFD shape and the trip-length separately, which makes four cases in total: the estimated MFD with $a_{1}=0$ (initial calibration), or with $a_{1}=0.8$ (re-calibration), and the estimated average trip length when $a_{1}=0$ (estimation by regression $L_{\mathrm{MFD}}$ ), or $a_{1}=$ 0.8 (the estimation from the mesoscopic simulation results $L_{\text {simul }}$ ). These calibration cases are summarized in Table 1 . The results for these four calibration options are presented in Figures 8(a) and (b) for the free-flow and congested cases respectively. It appears that the reservoir model fails to reproduce the mean steady state value given by the mesoscopic simulator if no re-calibration is performed on the MFD and the average trip length. On the other hand, the steady state is properly estimated when the two components of the model are fully re-calibrated. This demonstrates how sensitive the reservoir model is to local changes in demand distribution, which requires re-calibration. Figure 8(a) shows that the improvements gained by re-calibrating the trip length only are much better than re-calibrating the MFD only for this case. This suggests that the average trip length is indeed a key parameter that should be updated as soon as the mobility pattern changes in the reservoir. This can be seen in Figure 6(a), where $L_{\text {simul }}$ varies greatly with respect to $a_{1}$, whereas the free-flow part of the MFD is not really influenced by $a_{1}$; see also Figure $5(\mathrm{a})$. The opposite observation can be made for the congested case; see Figure 8(b). The main improvement comes from the MFD calibration. This can be explained when looking at Figure 5(a) and 6(a). The trip length is less sensitive than the MFD to $a_{1}$ for congested situations. A synthesis of the improvements due to the different possible calibration methods is presented in Table 2 .

Table 1. The four calibration cases for the MFD model

\begin{tabular}{rcccc}
\hline Calibration case & no calibration & MFD calibration & $L$ calibration & both calibrations \\
\hline MFD used & $\operatorname{MFD}\left(a_{1}=0\right)$ & $\operatorname{MFD}\left(a_{1}\right)$ & $\operatorname{MFD}\left(a_{1}=0\right)$ & $\operatorname{MFD}\left(a_{1}\right)$ \\
Trip length used & $L_{\mathrm{MFD}}\left(a_{1}=0\right)$ & $L_{\operatorname{MFD}}\left(a_{1}=0\right)$ & $L_{\text {simul }}\left(a_{1}\right)$ & $L_{\text {simul }}\left(a_{1}\right)$ \\
\hline
\end{tabular}

(a)

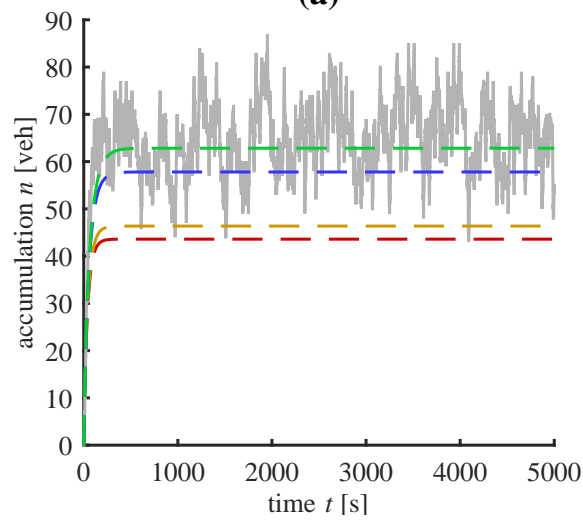

(b)

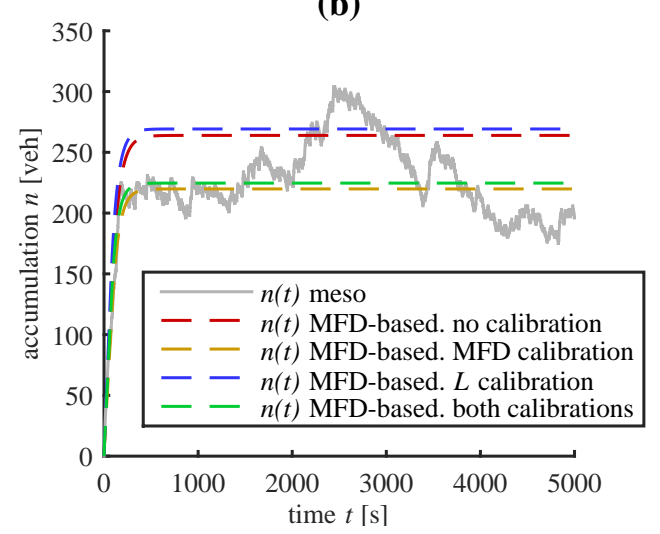

Figure 8. Comparison between the mesoscopic and the MFD-based framework when $a_{1}=0.8$ and $a_{2}=0-$ SC1. Different calibrations are considered for the MFD-based model: MFD and/or mean trip length calibrated from data corresponding to $a_{1}=0$ or $a_{1}=0.8$. (a) Evolution of accumulation $n(t)$ in free-flow, and (b) evolution of accumulation $n(t)$ in congested conditions 
It should be noted that the relative error on the steady state accumulation predicted by the reservoir model can be directly calculated for a given MFD, $L$ and the boundary conditions $\left(Q_{\mathrm{in}}^{D} ; Q_{\mathrm{out}}^{S}\right)$. In fact, the steady state related to equation 1 can be analytically determined without resorting to simulation. We use equation 2 to estimate the error propagation in the reservoir model. Assuming that there is an absolute error $\Delta P$ on the steady state production $P$ and an absolute error $\Delta L$ on the average trip length $L$, the absolute error $\Delta O$ on the steady state outflow $O$ is:

$$
\Delta O=\frac{1}{L} \Delta P-\frac{O}{L} \Delta L
$$

This error is related to the absolute error $\Delta n$ on the steady state accumulation $n$ via the derivative $O^{\prime}(n)$ of the outflow function, which satisfies $\left|O^{\prime}(n)\right|=\Delta O / \Delta n$ in the first approximation. Then, for the free-flow situations, we can neglect $\Delta P$ and estimate $\Delta L$ at $100 \mathrm{~m}$ by referring to Figures $5(\mathrm{a})$ and $6(\mathrm{a})$. In free-flow steady state, the outflow value should be $O=Q_{\mathrm{in}}^{D}=1.2 \mathrm{veh} / \mathrm{s}$, and the value of $L$ corresponds to $L_{\text {simul }}=515 \mathrm{~m}$ given by the mesoscopic simulation, see Figure $6(\mathrm{a})$. The derivative $O^{\prime}(n)=P^{\prime}(n) / L=0.012 \mathrm{~s}^{-1}$ is determined from the MFD plot for $n=n^{\text {meso }}=$ 66 veh in Figure 5(a). As a result, the relative error $\Delta n / n^{\text {meso }}$ from the mesoscopic approach can be roughly estimated at $30 \%$, which corresponds quite well to the error given in Table 2 when the MFD-based model is not re-calibrated. Similarly, for the congested situation, we can estimate $\Delta P=230 \mathrm{veh} . \mathrm{m} / \mathrm{s}$ for $n=n^{\text {meso }}=232 \mathrm{veh}$, and neglect $\Delta L$. We obtain $L=L_{\text {simul }}=370 \mathrm{~m}$ in Figure $6(\mathrm{a})$ and $\left|O^{\prime}(n)\right|=0.016 \mathrm{veh} / \mathrm{s}$ in Figure 5(a). Thus, the relative error $\Delta n / n^{\text {meso }}$ is estimated at $16 \%$, which again provides a good prediction of the error given in Table 2 for congestion situations and the initial MFD-based model setting.

Table 2. Comparison of accumulation values in steady state between the mesoscopic and the MFD-based approaches for scenario SC1 $\left(a_{1}=0.8\right.$ and $\left.a_{2}=0\right)$. Lowest errors are indicated in bold face

\begin{tabular}{cccccc}
\hline \multirow{2}{*}{ value } & mesoscopic & \multicolumn{3}{c}{ MFD-based } \\
\cline { 3 - 6 } & & no calibration & MFD calibration & $L$ calibration & both calibrations \\
\hline$n$ (free-flow) [veh] & 66 & 44 & 46 & $\mathbf{5 8}$ & $\mathbf{6 3}$ \\
error from meso [\%] & 0 & 33 & 29 & $\mathbf{1 2}$ & $\mathbf{4}$ \\
\hline$n$ (congestion) [veh] & 232 & 264 & $\mathbf{2 2 0}$ & 269 & $\mathbf{2 2 5}$ \\
error from meso [\%] & 0 & 14 & $\mathbf{5}$ & 16 & $\mathbf{3}$ \\
\hline
\end{tabular}

We now present the evolution of MFD-based errors for different heterogeneity coefficients varying from $a_{1}=0$ to 0.8 when $a_{2}=0$. The same free-flow and congested situations are studied, as described in section 3.2.1. The relative errors are calculated for the steady state accumulation, and now also for the outflow. Note that the inflows and outflows have the same values in steady-state. The steady state outflow is estimated by calculating the mean slope of the exit count curve $N_{\text {out }}(t)$ over the analysis period $[1000 ; 5000 \mathrm{~s}]$.

Figure 9 shows the relative errors in the MFD model in comparison with the mesoscopic one. It can be seen clearly that the higher the heterogeneity coefficient is, the higher the relative error. These results above all confirm that an accurate estimation of the mean trip length is essential in free-flow to obtain good simulation results from the MFD-based approach; see Figure 9(a). On the contrary, re-calibrating the MFD shape is crucial during congestion, see Figure 9(b). If the MFD shape and the mean 
trip length are not re-calibrated when the demand or supply distribution changes at the reservoir boundaries, simulation errors up to $30 \%$ can be observed. When recalibration is performed for these two key elements, the errors fall below $5 \%$ for all levels of heterogeneity.

Figures 9(c) and (d) show very different patterns when considering outflow errors. They appear to be independent of the calibration methods. This is because the total flow in steady state is completely defined by the global demand $Q_{\text {in }}^{D}$ in free-flow, and the global supply $Q_{\text {out }}^{S}$ in congestion. Thus we would have expected a perfect estimation of the in- and outflows in steady state using the MFD-based approach whatever the calibration method applied. However, some small discrepancies (around or less than 5\%) can still be observed in Figures 9(c) and (d). These are due to numerical approximations in the mesoscopic framework as the outflows are discretized in vehicle units.
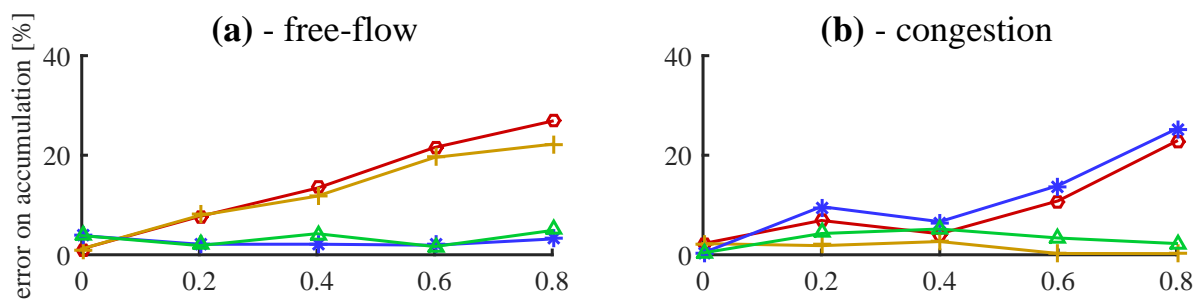

(c) - free-flow
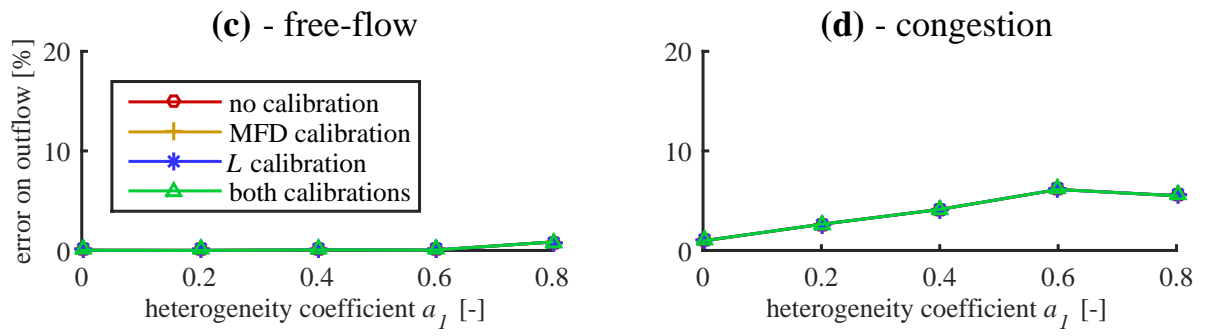

Figure 9. Relative comparison of the MFD-based and the mesoscopic simulation results for SC1 $\left(a_{2}=0\right)$. (a) Relative errors for steady state accumulation $n$ for different heterogeneity levels, in free-flow and (b) in congestion. (c) Relative errors for steady state outflow $Q_{\text {out }}$ for different heterogeneity levels, in free-flow and (d) in congestion.

\subsubsection{Comparison between mesoscopic and MFD-based approaches for homogeneous demand and heterogeneous supply loadings - scenario SC2}

We now focus on free-flow and congested network loadings with homogeneous demand distribution $a_{1}=0$ and heterogeneous supply distributions $a_{2}=0.6$, i.e. scenario $\mathrm{SC}$. The total demand and supply values are the same as in section 3.2.2. As in the previous section, mesoscopic and MFD-based simulation results are compared when the MFD and the mean trip length are re-calibrated or not with respect to the fully homogeneous case $a_{1}=0$ and $a_{2}=0$.

Figure 10 presents the evolution of accumulation for the mesoscopic and the MFDbased simulations with the four calibration cases. Figure 10(a) shows the results in the free-flow situation. It appears that the different calibration methods have no effect on the MFD-based simulation outputs, and that the steady state accumulation is rather well estimated in this case. This is not surprising because heterogeneous supply distributions only affect network loadings when exits act as bottlenecks, which is not the case in free-flow. This is confirmed by Figures 5(b) and 6(b), where neither the 
free-flow part of the MFD nor the estimated average trip length in free-flow depend on the heterogeneity coefficient $a_{2}$. The results are different in the congested situation; see Figure 10(b). The same conclusions are observed as in scenario SC1: re-calibrating the MFD allows reducing the error when estimating the steady state accumulation. The re-calibration of the average trip length has little impact in this case. This can be explained by the differences observed between cases $a_{2}=0$ and $a_{2}=0.6$ in Figure 5(b) for the MFD, and in Figure 6(b) for the mean trip length. A synthesis of the errors in steady state for SC2 is proposed in Table 3.

(a)

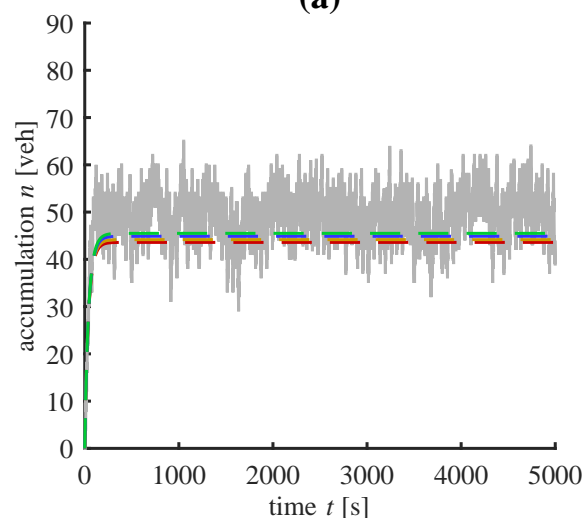

(b)

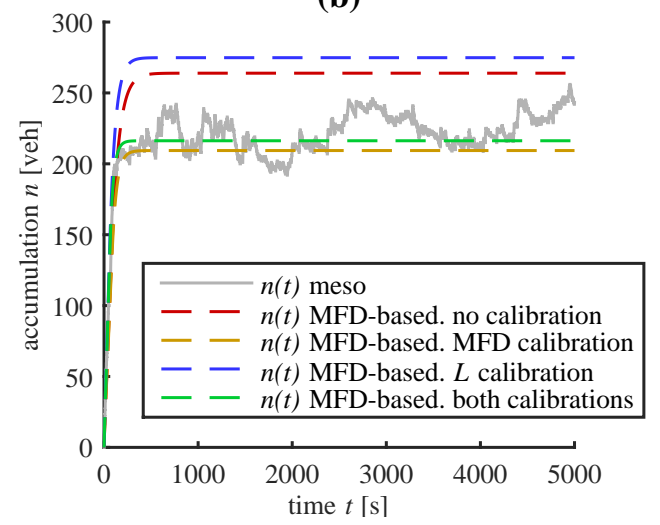

Figure 10. Comparison between the mesoscopic and the MFD-based approaches for SC2 $\left(a_{1}=0\right.$ and $a_{2}=$ 0.6). (a) Evolution of accumulation $n(t)$ in free-flow, and (b) evolution of accumulation $n(t)$ in congestion

Table 3. Comparison of accumulation values in steady state between the mesoscopic and the MFD-based frameworks for scenario SC2 $\left(a_{1}=0\right.$ and $\left.a_{2}=0.6\right)$. Lowest errors are indicated in bold face

\begin{tabular}{cccccc}
\hline \multirow{2}{*}{ value } & mesoscopic & \multicolumn{3}{c}{ MFD-based } \\
\cline { 3 - 6 } & & no calibration & MFD calibration & $L$ calibration & both calibrations \\
\hline$n$ (free-flow) [veh] & 49 & $\mathbf{4 3}$ & $\mathbf{4 4}$ & $\mathbf{4 5}$ & $\mathbf{4 6}$ \\
error from meso [\%] & 0 & $\mathbf{1 1}$ & $\mathbf{1 0}$ & $\mathbf{8}$ & $\mathbf{7}$ \\
\hline$n$ (congestion) [veh] & 223 & 264 & $\mathbf{2 0 9}$ & $\mathbf{2 1 6}$ \\
error from meso [\%] & 0 & 18 & $\mathbf{6}$ & 23 & $\mathbf{3}$ \\
\hline
\end{tabular}

As in section 3.2.2, we now present the evolution of MFD-based errors for different heterogeneity coefficients varying from $a_{2}=0$ to 0.6 when $a_{1}=0$.

Figure 11 shows the relative errors in the MFD model in comparison with the mesoscopic one. The trends oppose each other as in scenario SC1 when considering freeflow and congested situations. In congestion, errors may increase up to $30 \%$ and almost vanish when proper calibrations of both MFD and mean trip length are performed; see Figure 11(b). In free-flow, Figure 11(a) shows that there is almost no observable error because the heterogeneous supply distribution has no impact in this case.

As in SC1, almost no errors are observed for the outflow in free-flow conditions, see Figure 11(c). This is no longer the case for congestion as huge errors that increase with the heterogeneity coefficient (almost up to 150\%) are observed in Figure 11(d). The phenomena behind this unexpected result will be described extensively in the next section. In brief, discrepancies between the mesoscopic and the MFD models can be explained by congestion patterns that propagate within the reservoir in the mesoscopic simulation. The total outflow $Q_{\text {out }}$ from the network falls below the global 
supply limitation $Q_{\text {out }}^{S}$ in the mesoscopic outputs, while $Q_{\text {out }}$ remains exactly equal to $Q_{\mathrm{out}}^{S}$ in the MFD-based approach. The mesoscopic simulator is able to account for congestion spillbacks between internal intersections that induce flow reduction at several local exits. This phenomenon is not reproduced by the MFD-based approach as the perimeter flow is always considered as uniform. In fact, this phenomenon also influences the accumulation values in steady states, but this is hardly visible in Figure 11(b) as the differences in accumulation are relatively small for severe congestion. Moreover, the range of accumulation errors is of the same magnitude as the variability of the $n(t)$ outputs from the mesoscopic simulation. Unlike in SC1, this shows that, while being sufficient to accurately estimate the mean steady state accumulation, the proper calibration of the reservoir model (MFD shape and mean trip length) cannot ensure a good estimation of steady state flow in congestion.
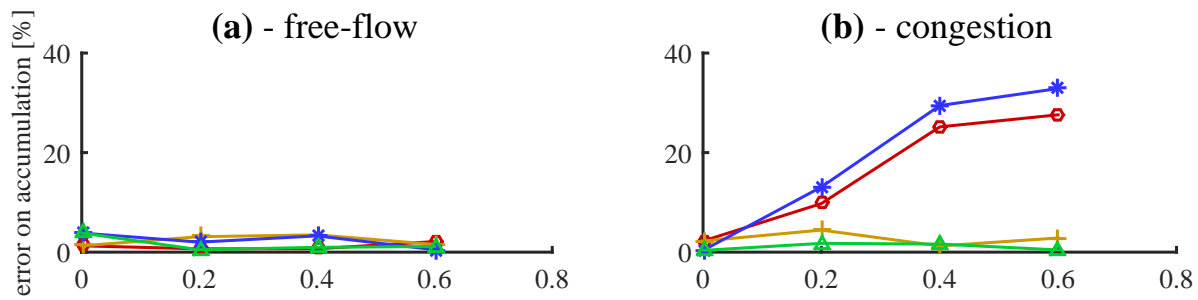

(c) - free-flow
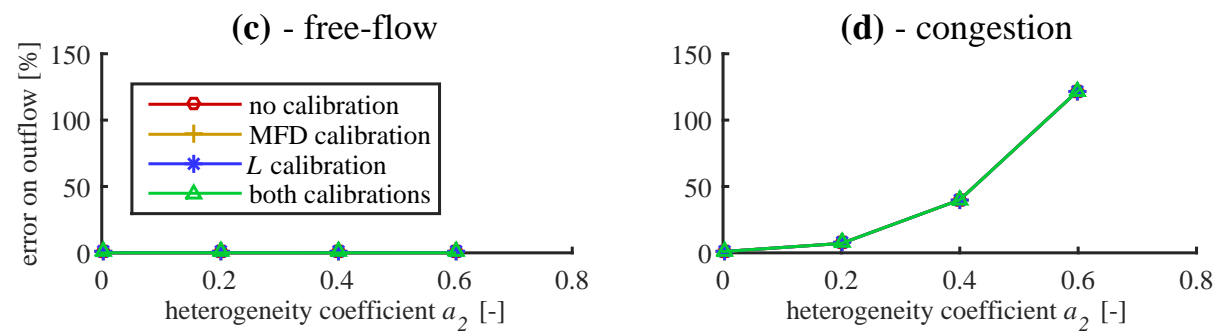

Figure 11. Relative comparison of the MFD-based and the mesoscopic simulation results for SC2 $\left(a_{1}=0\right)$. (a) Relative errors for steady state accumulation $n$ for different heterogeneity levels, in free-flow and (b) in congestion. (c) Relative errors for steady state outflow $Q_{\text {out }}$ for different heterogeneity levels, in free-flow and (d) in congestion.

\subsubsection{Studying drops in total outflow due to internal congestion patterns}

This section investigates in detail the congestion patterns that appear within the reservoir when using the mescoscopic simulator. We notably aim to propose a correction method capable of reducing the discrepancies observed in the outflow for the MFD simulation and that cannot be improved by simply re-calibrating the MFD or the trip length.

Let us now focus on the evolution of the global outflow $Q_{\text {out }}(t)$ given by the mesoscopic simulator for different supply values at the exits. Both scenarios SC1 and SC2 are studied. Figure 12 shows the simulation results when the total demand $Q_{\text {in }}^{D}$ is equal to $3 \mathrm{veh} / \mathrm{s}$ and $Q_{\text {out }}^{S}=0.6$ to $6 \mathrm{veh} / \mathrm{s}$. Figure 12 (a) shows the results for SC1, and Figure 12(b) the results for SC2. In these figures, one point corresponds to the steady state global outflow $Q_{\text {out }}$ obtained in the mesoscopic simulation for a given global supply $Q_{\text {out }}^{S}$. Two reference lines have been added to the graphs: the MFD capacity (maximum outflow) obtained with homogeneous boundary conditions (SC0) and the first bisector that corresponds to total outflow equal to the total supply. Two very distinct situations are observed for scenarios SC1 and SC2. For SC1, the steady state 
outflow matches either the network capacity (gray area in the graph) or the available total supply $Q_{\text {out }}^{S}$. It should be recalled that the network capacity depends on the heterogeneity coefficient $a_{1}$ in SC1, see Figure 5(a). For SC2, the total outflow starts to decrease well before the supply becomes a constraint at the aggregated level. Furthermore, the difference between the total outflow and the available total supply increases with the level of heterogeneity. As already mentioned, this is the result of internal and local congestion patterns with spillbacks between intersections that reduce the local outflows even below the available local capacity.

(a)

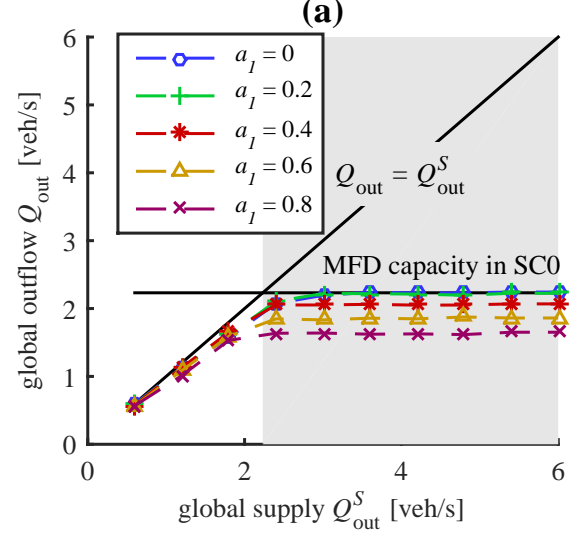

(b)

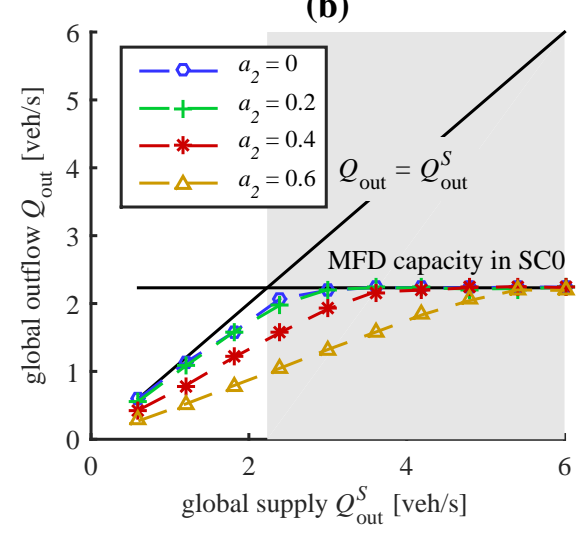

Figure 12. Steady state total outflow versus total supply in the mesoscopic framework. (a) Results for scenario SC1 (heterogeneous demand distribution), and (b) scenario SC2 (heterogeneous supply distribution).

A simulation example including internal congestion patterns that leads to a drop in outflow is presented in Figure 13. The $N$-curves corresponds to scenario SC2 with $a_{2}=0.6$. In the MFD-based modeling it can be seen that the steady state outflow corresponding to the $N$-curve slopes is $Q_{\text {out }}^{S}=0.6 \mathrm{veh} / \mathrm{s}$. However, the total outflow starts decreasing after $t=1500 \mathrm{~s}$ in the mesoscopic simulator, and then stabilizes to a value of almost half of the total supply. This can be explained as follows. At the link level, due to heterogeneity in supply distribution, only a small fraction of vehicles can exit through D2; see Figure 2(c). This makes the route O2-D2 increasingly congested. Before the congestion propagates through the whole route, the outflow still corresponds to the maximum flow allowed for each exit. This is the situation at time $t_{1}$ for instance, for which the network state (density in each link) is described in figure 13(b). However, after a certain time (around $1500 \mathrm{~s}$ ) route O2-D2 becomes fully congested. This makes all the users go to destinations other than D2, but travel on at least one link of the arterial O2-D2, thus exiting the network at a lower rate. As a result, the other exits D1 and D3 to D6 do not function at full capacity; see time $t_{2}$ in Figure 13(c). As a result, the total steady state outflow is below the sum of all the exit capacities.

We further analyze this internal congestion pattern to implement a possible correction in the MFD-based model. We observe that such a pattern is actually characterized by two parameters: the time $T_{c}$ at which a slope break appears in $N_{\text {out }}(t)$, and the new value of the slope, i.e. the effective global outflow $Q_{\text {out }}^{\text {eff }}$, see figure 14(c). Note that this new value defines the steady-state situation. Several simulations were run in scenario SC2 with $a_{2}=0$ to 0.9 and $Q_{\text {out }}^{S}=0.6$ to $3 \mathrm{veh} / \mathrm{s}$ to study the evolution of these two parameters. The results are presented in Figure 14(a) for $T_{c}$ and Figure 14(b) for $Q_{\text {out }}^{\text {eff }}$. The results for $T_{c}$ are only given for $Q_{\text {out }}^{S}$ lower than $1.2 \mathrm{veh} / \mathrm{s}$. This is because as the supply value increases, the drop in the total outflow occurs near $t=0$ during the warm-up period, before the first equilibrium state has been reached. Thus for $Q_{\text {out }}^{S}>$ 
(a)

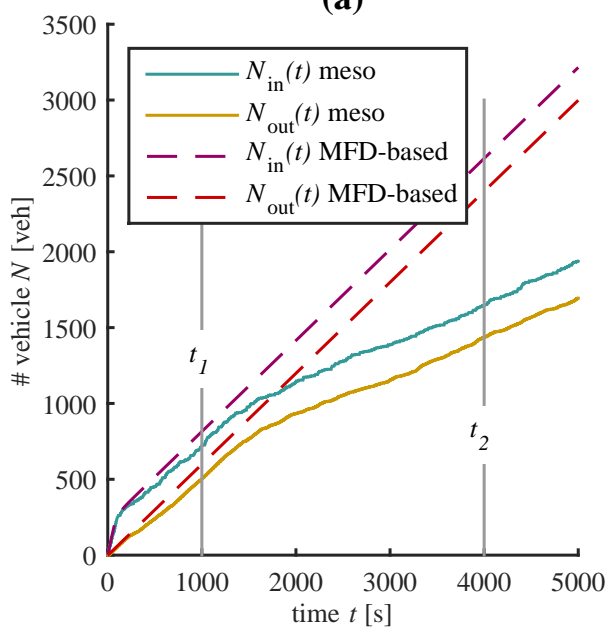

(b) $-t_{1}$

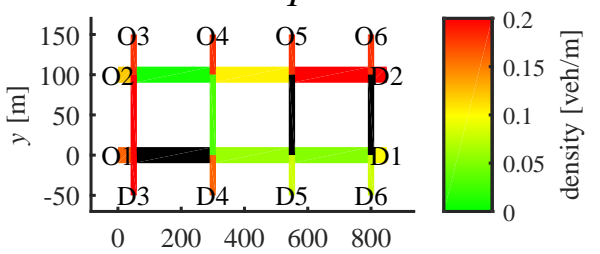

(c) $-t_{2}$

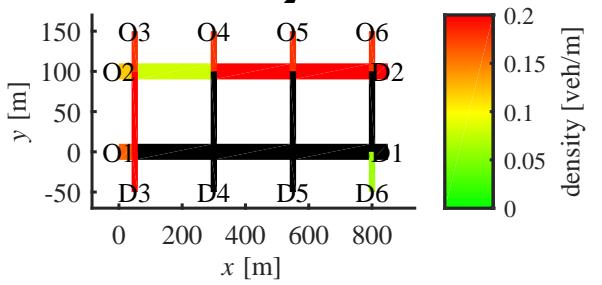

Figure 13. An example of outflow drop due to a local internal congestion pattern in the mesoscopic framework - SC2 with $a_{2}=0.6$. (a) $N$-curves calculated by the mesoscopic and the MFD-based approaches, (b) traffic states at the link-level (density on each link calculated with the mesoscopic simulator, the black color meaning null density) at time $t_{1}$, and (c) at time $t_{2}$.

$1.2 \mathrm{veh} / \mathrm{s}$ we can consider that $T_{c}$ is equal to 0 .

When a drop in outflow is actually observed, we find that the two parameters $T_{c}$ and $Q_{\text {out }}^{\text {eff }}$ may be well approximated by a bilinear function of the heterogeneity coefficient $a_{2}$ and the global supply $Q_{\mathrm{out}}^{S}$ :

$$
T_{c}\left(a_{2}, Q_{\mathrm{out}}^{S}\right)= \begin{cases}\left(\alpha_{t} Q_{\mathrm{out}}^{S}+\beta_{t}\right) a_{2}+\gamma_{t} Q_{\mathrm{out}}^{S}+\delta_{t} & \text { if } a_{2} \geq 0.2 \text { and } Q_{\mathrm{out}}^{S} \leq 1.2 \mathrm{veh} / \mathrm{s} \\ 0 & \text { otherwise }\end{cases}
$$

$Q_{\text {out }}^{\mathrm{eff}}\left(a_{2}, Q_{\mathrm{out}}^{S}\right)=\left(\alpha_{q} Q_{\mathrm{out}}^{S}+\beta_{q}\right) a_{2}+\gamma_{q} Q_{\mathrm{out}}^{S}+\delta_{q}$

where $\alpha_{t}=1.8 \times 10^{3} \mathrm{veh}^{-1} . \mathrm{s}^{2}, \beta_{t}=-4.2 \times 10^{3} \mathrm{~s}, \gamma_{t}=-2.1 \times 10^{3} \mathrm{veh}^{-1} . \mathrm{s}^{2}, \beta_{t}=4.9 \times 10^{3}$ $\mathrm{s}, \alpha_{q}=-1.1, \beta_{q}=0.071 \mathrm{veh} / \mathrm{s}, \gamma_{q}=1.1$, and $\delta_{q}=-0.051 \mathrm{veh} / \mathrm{s}$. Note that the fit for $Q_{\text {out }}^{\mathrm{eff}}$ is still consistent when $a_{2}=0$ (scenario SC0), as the bilinear function gives $Q_{\mathrm{out}}^{\mathrm{eff}}$ almost equal to $Q_{\text {out }}^{S}$.

As we now have a complete description of the effect of the congestion pattern on outflow, it is easy to implement a correction method in the MFD-based model to account for the outflow limitation $Q_{\text {out }}^{\text {eff }}\left(a_{2}, Q_{\text {out }}^{S}\right)$ after the time $T_{c}\left(a_{2}, Q_{\text {out }}^{S}\right)$. The result of this correction method is presented in Figure 14(d) for the same example we analyzed in Figure 13(a). It shows that the correction method greatly improves the MFD-based results compared to Figure 13(a). 
(a)

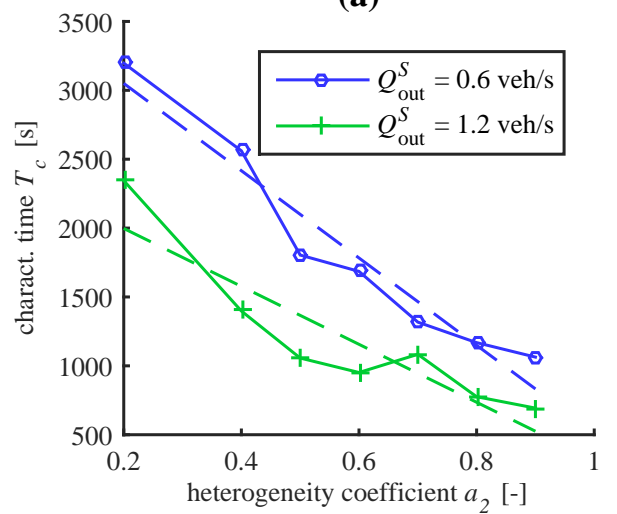

(c)

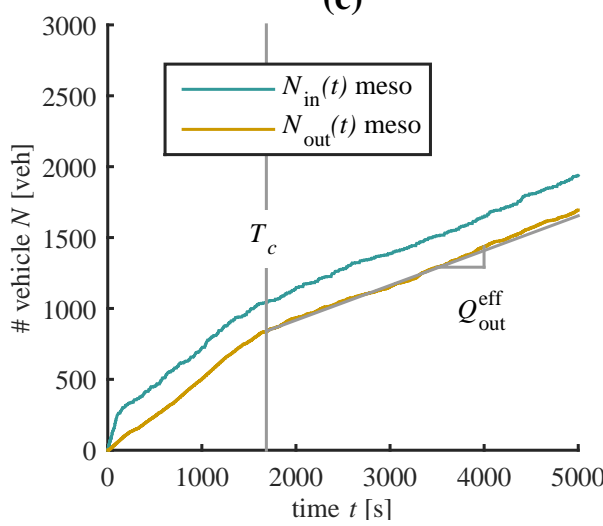

(b)

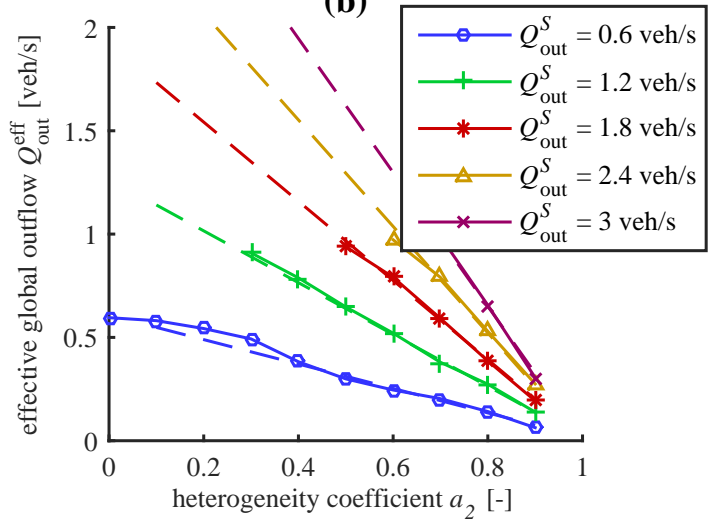

(d)

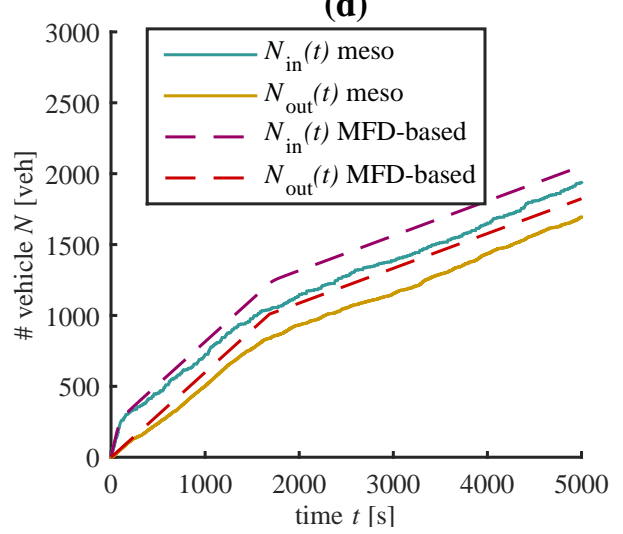

Figure 14. (a) Evolution of time $T_{c}$ when an outflow drop is observed in the mesoscopic simulation, for different heterogeneity levels $a_{2}$ in scenario SC2 and different supply values $Q_{\text {out }}^{S}$. (b) Evolution of the effective steady state total outflow $Q_{\mathrm{out}}^{\mathrm{eff}}$ after the outflow drop in the mesoscopic simulation, for different $a_{2}$ and $Q_{\mathrm{out}}^{S}$. (c) Definition of $T_{c}$ and $Q_{\text {out }}^{\text {eff }}$ on the mesoscopic $N$-curves. (d) Application of the correction method based on $T_{c}$ and $Q_{\mathrm{out}}^{\text {eff }}$ to the MFD-based approach 


\section{Discussion}

In this study, we compared steady state simulation results for an MFD-based model and a mesoscopic model when considering a simple network. The MFD-based model describes traffic states at the aggregated (reservoir) level, while the mesoscopic tool provides a complete description of traffic dynamics at the link level inside the reservoir. The purpose of such a comparison was to test the robustness of the aggregated approach to different heterogeneous loadings at the network perimeter. Two heterogeneous scenarios were considered. SC1 assumes a heterogeneous demand distribution at the network entries, while the supply distribution is homogeneous. The reverse case, i.e. SC2, considers a heterogeneous supply distribution at the network exits, while the demand distribution is homogeneous. The results on the network studied showed that heterogeneity coefficients $a_{1}$ and $a_{2}$ have a strong impact on its MFD shape and its average trip length. While such impacts may not be universally expected for other network configurations, we showed that the proper calibration of these two crucial features of the MFD-based approach is essential to avoid errors (up to $30 \%$ in our case study) when predicting steady traffic states.

More interestingly and more generally, we highlighted a major difference between the two scenarios. In SC1, a proper re-calibration of the MFD and the mean trip length allows reducing the prediction errors completely (down to 5\%). However, in SC2, the appearance of internal congestion patterns including spillbacks between intersections close to the reservoir perimeter makes the re-calibration almost inefficient. In this case, the MFD-based model fails to reproduce the network's internal dynamics. In particular, we noticed significant drops in the total outflow in the mesoscopic outputs that were not reproduced by the MFD-based approach. Analyzing congestion patterns with spillbacks using the mesoscopic simulator makes it possible to propose a correction method to account for the outflow drop in the MFD-based model.

With our simple configuration, it should be kept in mind that the congestion observed with highly heterogeneous supply values is emphasized by the constraints of our simple network, i.e. the users are forced to exit through a specific link. In a context of a multi-reservoir system, a dense and well-connected grid network would offer several re-routing options to avoid local congestion at the exit border of a reservoir. If such a border has many connections to the downstream reservoirs, the spillbacks and resulting outflow drops observed may be less severe in reality. Nevertheless, sparse connectivity between reservoirs is very likely to be observed in European type cities, in the case of bridges or major arterials that connect different urban areas. In such a configuration, the users are forced to exit through a specific connection point in their trip, unless major re-routing is imposed. Our study is specifically intended to question the robustness of MFD-based simulation when the capacities of such critical connection points are heterogeneously distributed along the reservoir border.

\subsection{Study of a bigger network}

As one of the most critical limitation of the present work is the relatively small size of the network, and thus the lack of redundancy and routing options, we provide additional results for a bigger grid network with the same kind of configuration, see Figure 15. This new topology consists of a $6 \times 6$ one-way Manhattan network with West-East links more than twice as long as North-South ones. The same scenarios SC1 and SC2 have been applied to this network. Figure 16 shows clearly that the 
various boundary settings significantly influence the MFD shape. Nevertheless, a lesser influence on the trip length is noticed in this case of a more redundant network: the order of magnitude of its variations in free-flow is only around $7 \%$ for $\mathrm{SC} 1$.

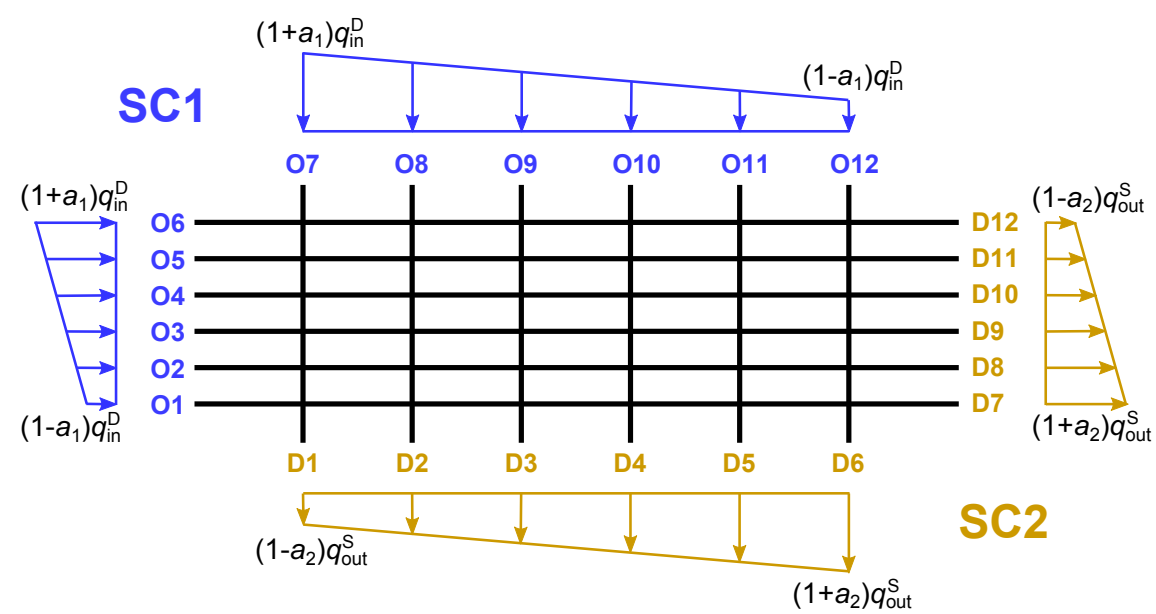

Figure 15. New network configuration with scenarios SC1 and SC2 settings
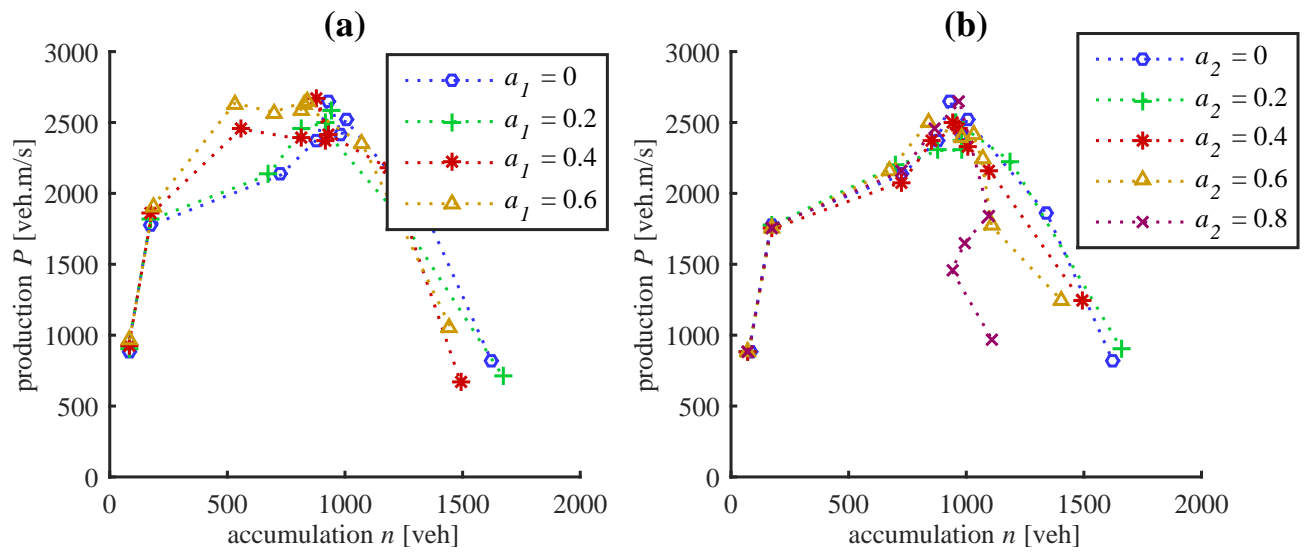

(c)
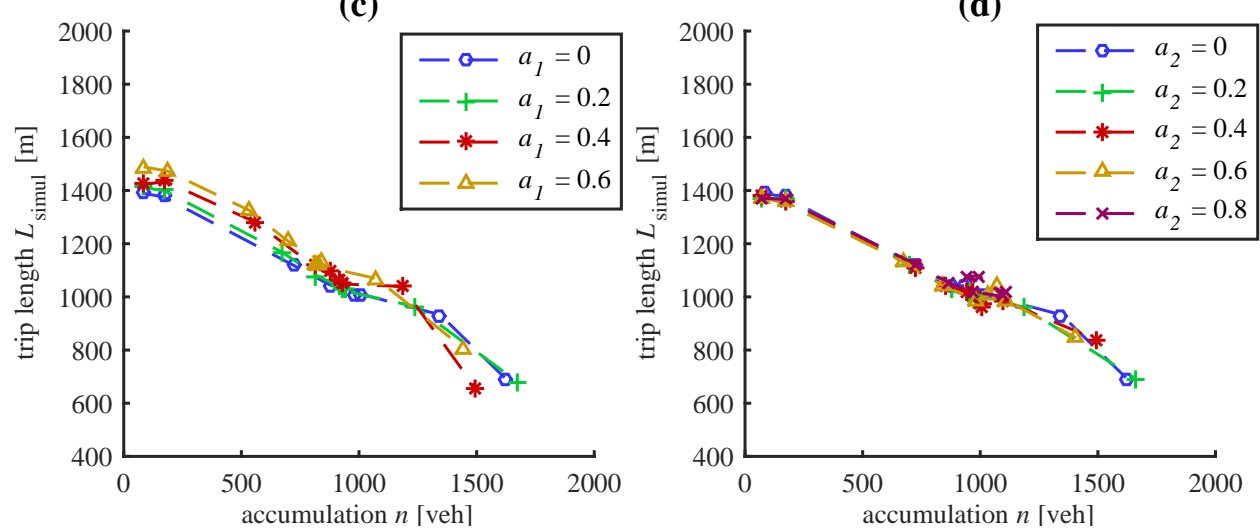

Figure 16. (a) Network MFD for different coefficients $a_{1}$ in SC1 and (b) for different $a_{2}$ in SC2; (c) vehicle average trip length for different coefficients $a_{1}$ in SC1 and (d) for different $a_{2}$ in SC2

The relative errors in the MFD model compared with the mesoscopic simulations 
are presented in Figure 17 for SC1 and in Figure 18 for SC2. Regarding the steady state accumulation, both figures show that the errors can be reduced to acceptable values once the MFD and the trip length are well calibrated. Similar conclusions are found with this new network in free-flow, i.e. re-calibrating the trip length only improves the MFD simulation results much more than re-calibrating the MFD only. Regarding the steady state outflow, we observed huge errors for congested situations in both scenarios. In SC2, these errors can be explained by outflow drop phenomena, similar to what we observed in the $2 \times 4$ network, as Figure 19 (b) suggests. However, the major difference with this $6 \times 6$ network is the significant error (more than $20 \%$ ) in outflow made for the homogeneous case $\left(a_{1}=a_{2}=0\right)$. This is also due to an outflow drop, independent of the heterogeneity coefficient, as presented in Figure 19(a). This means that even homogeneous loadings can trigger internal congestion patterns in this bigger network. Such an outflow drop phenomenon was in fact impossible to present with our smaller network. While this new mechanism should be further investigated, a simple outflow correction method can still be implemented in the reservoir MFD model to account for it. Note that such a correction does not depend on the boundary settings in SC1, therefore the total effective outflow $Q_{\text {out eff can be estimated one for }}$ all as shown in figure 19(a), and then implemented in the MFD model to modify the outflow $Q_{\text {out }}$ during congestion. This is completely different from the heterogeneous supply case (SC2), where the correction method we propose actually depends on the boundary settings, see section 3.2.4.
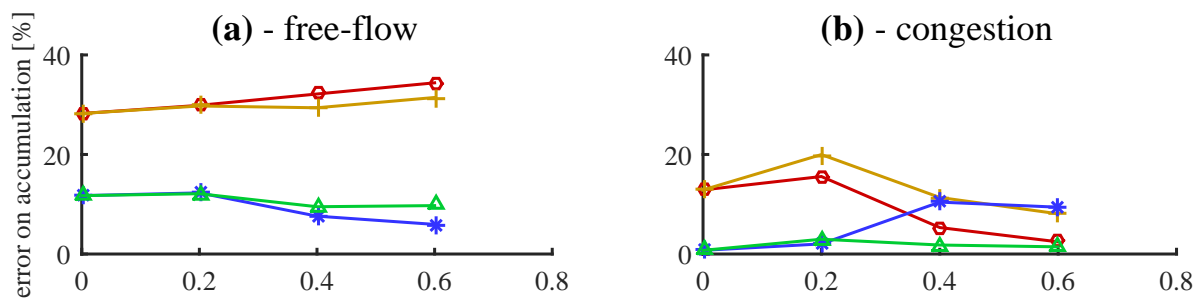

(c) - free-flow
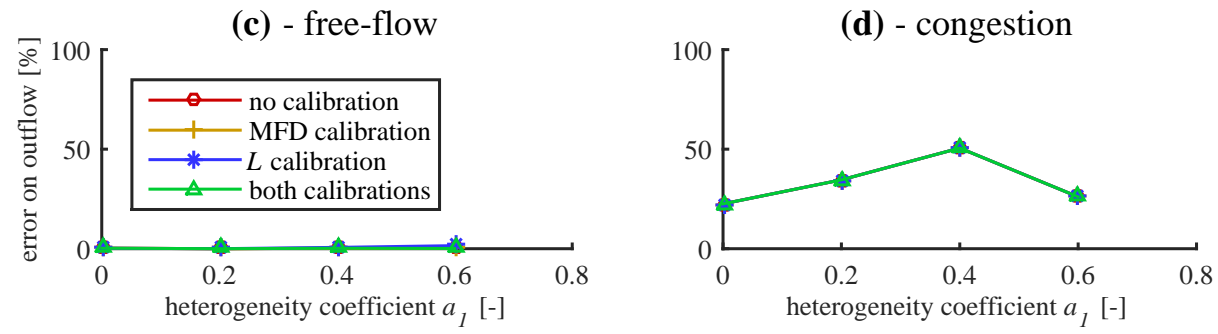

Figure 17. Relative comparison of the MFD-based and the mesoscopic simulation results for SC1 ( $a_{2}=0$ ). (a) Relative errors for steady state accumulation $n$ for different heterogeneity levels in free-flow and (b) in congestion. (c) Relative errors for steady state outflow $Q_{\text {out }}$ for different heterogeneity levels in free-flow and (d) in congestion.

\subsection{General conclusion}

While being specific to the kind of grid networks studied here, the results of this paper permit highlighting possible shortcomings in MFD-based simulation due to heterogeneous link-level settings at reservoir boundaries. In particular, this paper clearly shows that unlike the hypothesis made in the seminal paper on MFD, the OD matrix and the flow distribution between local entries and exits may matter when studying traffic dynamics at the network level. Large changes for these elements are likely to require 

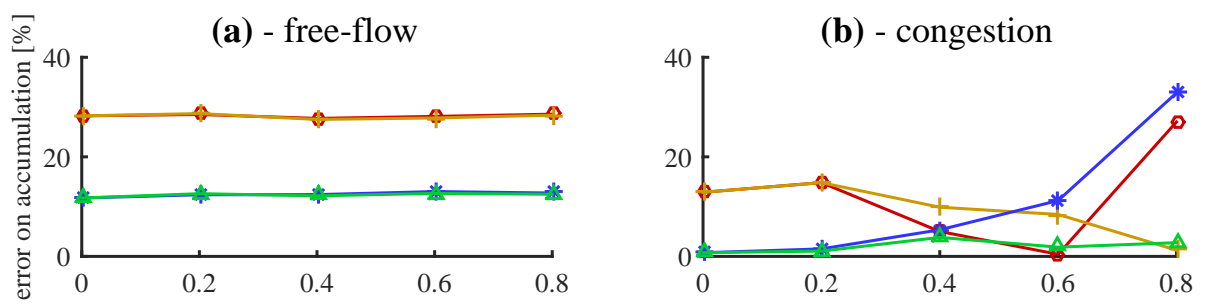

(c) - free-flow
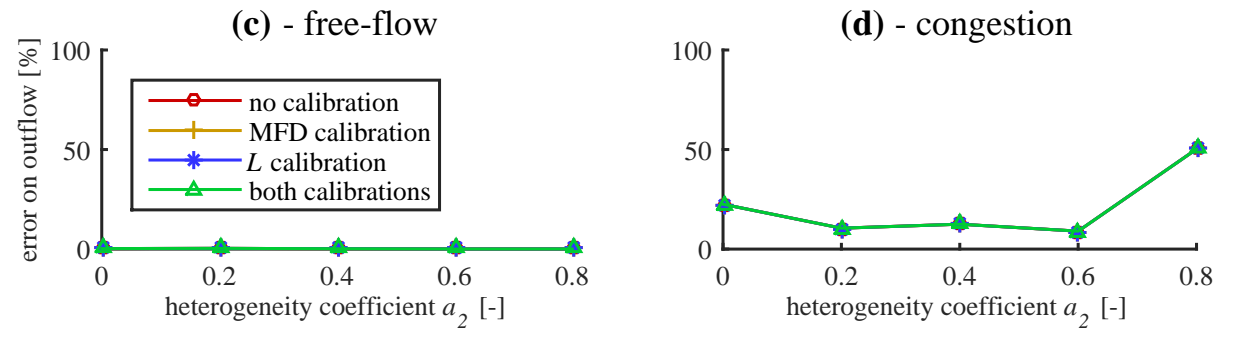

Figure 18. Relative comparison of the MFD-based and the mesoscopic simulation results for $\mathrm{SC} 2\left(a_{1}=0\right)$. (a) Relative errors for steady state accumulation $n$ for different heterogeneity levels in free-flow and (b) in congestion. (c) Relative errors for steady state outflow $Q_{\text {out }}$ for different heterogeneity levels in free-flow and (d) in congestion.

(a)

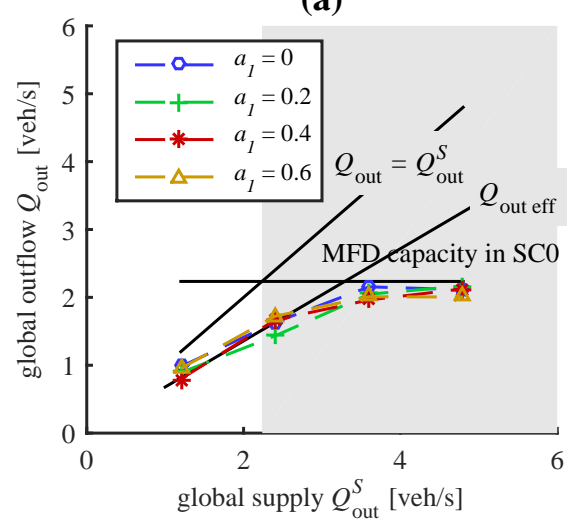

(b)

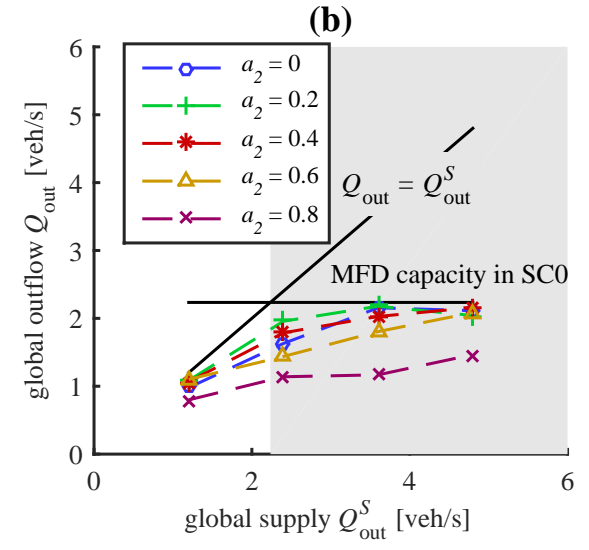

Figure 19. (a) Total effective outflow vs total exit supply for different coefficients $a_{1}$ in SC1 and (b) for different $a_{2}$ in SC2

re-calibrating both the MFD and the mean trip length, especially for irregular and asymmetrical networks. In practice, this is critical for applications based on the MFD like perimeter control, where the re-calibration should then be done on a regular time basis to account for changes in OD patterns. In further studies, it would be interesting to investigate the time scale of the re-calibrations, i.e. how often we should re-calibrate, and if both time scales for MFD and trip length are similar or not. One may still argue that the re-calibration could be avoided when directly integrating a level of uncertainty in the MFD, as this concept is notably studied in Gao and Gayah (2018) and in the robust control strategy from Haddad and Shraiber (2014). Nevertheless, our conclusions show that in some cases, even an accurate calibration may not be sufficient to reproduce the network dynamics well with the MFD-based model. More detailed knowledge of local capacity at exits, meaning a more detailed description of how congestion propagates from the links connected with the reservoir neighborhood, is required to catch the outflow drops that may occur. Note that this crucial issue has not been addressed when studying multi-reservoir systems (Yildirimoglu, Ramezani, 
and Geroliminis 2015) and thus will need further research. Interestingly, we showed that a simple correction method based on network observations in different configurations may be sufficient to update MFD-based simulation outputs.

Further research is needed to study the propagation of simulation errors in multireservoir systems to guaranty that such an approach can be a valid option for simulating large-scale urban areas and making traffic predictions. Efficient methods for re-calibrating the model in real time should also be investigated.

\section{Acknowledgements}

This project has received funding from the European Research Council (ERC) under the European Union's Horizon 2020 research and innovation program (grant agreement No 646592 - MAGnUM project).

\section{Disclosure statement}

No potential conflict of interest was reported by the authors.

\section{References}

Aboudolas, Konstantinos, and Nikolas Geroliminis. 2013. "Perimeter and boundary flow control in multi-reservoir heterogeneous networks." Transportation Research Part B: Methodological 55: 265-281. http://www.sciencedirect.com/science/article/pii/ S0191261513001185

Ampountolas, Konstantinos, Nan Zheng, and Nikolas Geroliminis. 2017. "Macroscopic modelling and robust control of bi-modal multi-region urban road networks." Transportation Research Part B: Methodological 104: 616-637. http://www.sciencedirect.com/science/ article/pii/S0191261515300370.

Barcelo, J., J. Casas, D. Garca, and J. Perarnau. 2006. "A Hybrid Simulation Framework for Advanced Transportation Analysis." \{IFAC\} Proceedings Volumes 39 (12): 497-502. 11th \{IFAC $\}$ Symposium on Control in Transportation Systems, http://www. sciencedirect. com/science/article/pii/S1474667015336715.

Batista, S. F. A., Ludovic Leclercq, and Nikolas Geroliminis. 2019. "Estimation of regional trip length distributions for the calibration of the aggregated network traffic models." Transportation Research Part B: Methodological 122: 192-217. http://www. sciencedirect.com/ science/article/pii/S0191261518311603.

Ben-Akiva, Moshe, Michel Bierlaire, Haris N. Koutsopoulos, and Rabi Mishalani. 2002. Real Time Simulation of Traffic Demand-Supply Interactions within DynaMIT, Vol. 63, Chap. 2, 19-36. Boston, MA: Springer US.

Buisson, Christine, and Cyril Ladier. 2009. "Exploring the impact of homogeneity of traffic measurements on the existence of Macroscopic Fundamental Diagrams." Transportation Research Record: Journal of the Transportation Research Board 2124: 127-136.

Burghout, Wilco, N Koutsopoulos, Haris, and Ingmar Andreasson. 2005. "Hybrid MesoscopicMicroscopic Traffic Simulation." Transportation Research Record: Journal of the Transportation Research Board 1934: 218-225.

Daganzo, Carlos F. 1994. "The cell transmission model: A dynamic representation of highway traffic consistent with the hydrodynamic theory." Transportation Research Part B: Methodological 28 (4): 269-287. http://www.sciencedirect.com/science/article/pii/ 0191261594900027. 
Daganzo, Carlos F. 2007. "Urban gridlock: Macroscopic modeling and mitigation approaches." Transportation Research Part B: Methodological 41 (1): 4962. http://www.sciencedirect.com/science/article/B6V99-4JWFGNC-2/1/ 4659a928f cc742053a9587464572f409.

Daganzo, Carlos F., and Nikolas Geroliminis. 2008. "An analytical approximation for the macroscopic fundamental diagram of urban traffic." Transportation Research Part B: Methodological 42 (9): 771-781. http://www.sciencedirect.com/science/article/ B6V99-4T4WKTN-1/2/f cb0886e1a027e33bf8e5eefc2105126.

de Jong, D., V.L. Knoop, and S.P. Hoogendoorn. 2013. "The effect of signal settings on the macroscopic fundamental diagram and its applicability in traffic signal driven perimeter control strategies." In Intelligent Transportation Systems - (ITSC), 2013 16th International IEEE Conference on, Oct, 1010-1015.

Doig, Jean C, Vikash V Gayah, and Michael J Cassidy. 2013. "Inhomogeneous Flow Patterns in Undersaturated Road Networks: Implications for Macroscopic Fundamental Diagram." Transportation Research Record: Journal of the Transportation Research Board 2390: 68-75.

Edie, L. C. 1963. "Discussion of traffic stream measurements and definitions." In 2nd International Symposium on the Theory of Traffic Flow, Paris, 139154.

Gao, Xueyu, and Vikash V. Gayah. 2018. "An analytical framework to model uncertainty in urban network dynamics using Macroscopic Fundamental Diagrams." Transportation Research Part B: Methodological 117: 660-675. TRB:ISTTT-22, http://www . sciencedirect . com/science/article/pii/S0191261517306884.

Gayah, Vikash V., and Carlos F. Daganzo. 2011. "Clockwise hysteresis loops in the Macroscopic Fundamental Diagram: An effect of network instability." Transportation Research Part B: Methodological 45 (4): 643-655. http: //www.sciencedirect.com/science/article/pii/ S0191261510001396.

Gayah, Vikash V., Xueyu Gao, and Andrew S. Nagle. 2014. "On the impacts of locally adaptive signal control on urban network stability and the Macroscopic Fundamental Diagram." Transportation Research Part B: Methodological 70: 255-268. http://www. sciencedirect. com/science/article/pii/S0191261514001647.

Geroliminis, Nikolas, and Carlos F Daganzo. 2007. "Macroscopic Modeling of Traffic in Cities." In Transportation Research Board 86th Annual Meeting, 07-0413, Washington DC.

Geroliminis, Nikolas, and Jie Sun. 2011a. "Hysteresis phenomena of a Macroscopic Fundamental Diagram in freeway networks." Transportation Research Part A: Policy and Practice 45 (9): 966-979. http://www.sciencedirect.com/science/article/pii/ S0965856411000620.

Geroliminis, Nikolas, and Jie Sun. 2011b. "Properties of a well-defined macroscopic fundamental diagram for urban traffic." Transportation Research Part B: Methodological 45 (3): 605-617. http://www.sciencedirect.com/science/article/pii/S0191261510001372.

Gipps, P.G. 1986. "Multsim: a model for simulating vehicular traffic on multi-lane arterial roads." Mathematics and Computers in Simulation 28 (4): 291-295. http://www. sciencedirect.com/science/article/pii/0378475486900509.

Girault, Jan-Torben, Vikash V Gayah, Ilgin Guler, and Monica Menendez. 2016. "Exploratory Analysis of Signal Coordination Impacts on Macroscopic Fundamental Diagram." Transportation Research Record: Journal of the Transportation Research Board 2560: 36-46.

Godfrey, J. W. 1969. "The mechanism of road network." Traffic Engineering and Control 7 (11): $323-327$.

Haddad, Jack, and Boris Mirkin. 2017. "Coordinated distributed adaptive perimeter control for large-scale urban road networks." Transportation Research Part C: Emerging Technologies 77: 495-515. http://www.sciencedirect.com/science/article/pii/ S0968090X16302509.

Haddad, Jack, and Arie Shraiber. 2014. "Robust perimeter control design for an urban region." Transportation Research Part B: Methodological 68: 315-332. http://www. sciencedirect. com/science/article/pii/S0191261514001179.

Hajiahmadi, M., V.L. Knoop, B. De Schutter, and H. Hellendoorn. 2013. "Optimal dynamic 
route guidance: A model predictive approach using the macroscopic fundamental diagram." In Intelligent Transportation Systems - (ITSC), 2013 16th International IEEE Conference on, Oct, 1022-1028.

Herman, R., and I. Prigogine. 1979. "A two-fluid approach to town traffic." Science 204 (4389): 148-151. https://www.scopus.com/inward/record.uri?eid=2-s2. $0-0000539272 \&$ partner ID $=40$ \&md5 $=f 7880946 \mathrm{~d} 206 \mathrm{a} 8 \mathrm{fdf} 886 \mathrm{baaf} 424208 \mathrm{ba}$.

Ji, Yuxuan, and Nikolas Geroliminis. 2012. "On the spatial partitioning of urban transportation networks." Transportation Research Part B: Methodological 46 (10): 1639-1656. http:// www.sciencedirect.com/science/article/pii/S0191261512001099.

Joueiai, Mahtab, Ludovic Leclercq, Hans Van Lint, and Serge P. Hoogendoorn. 2015. "Multiscale Traffic Flow Model Based on the Mesoscopic LighthillWhitham and Richards Models." Transportation Research Record: Journal of the Transportation Research Board 2491: 98106.

Keyvan-Ekbatani, M., R. C. Carlson, V. L. Knoop, S. P. Hoogendoorn, and M. Papageorgiou. 2016. "Queuing under perimeter control: Analysis and control strategy." In 2016 IEEE 19th International Conference on Intelligent Transportation Systems (ITSC), Nov, 1502-1507.

Keyvan-Ekbatani, Mehdi, Anastasios Kouvelas, Ioannis Papamichail, and Markos Papageorgiou. 2012. "Exploiting the fundamental diagram of urban networks for feedback-based gating." Transportation Research Part B: Methodological 46 (10): 1393-1403. http://www . sciencedirect.com/science/article/pii/S0191261512000926.

Keyvan-Ekbatani, Mehdi, Markos Papageorgiou, and Victor L. Knoop. 2015. "Controller design for gating traffic control in presence of time-delay in urban road networks." Transportation Research Part C: Emerging Technologies 59: 308-322. Special Issue on International Symposium on Transportation and Traffic Theory, http://www. sciencedirect. com/science/article/pii/S0968090X1500176X.

Keyvan-Ekbatani, Mehdi, Markos Papageorgiou, and Ioannis Papamichail. 2013. "Urban congestion gating control based on reduced operational network fundamental diagrams." Transportation Research Part C: Emerging Technologies 33: 74-87. http://www . sciencedirect. com/science/article/pii/S0968090X13000922.

Kim, Jiwon, and Hani S. Mahmassani. 2015. "Compound Gamma representation for modeling travel time variability in a traffic network." Transportation Research Part B: Methodological 80: 40-63. http://www.sciencedirect.com/science/article/pii/S019126151500140X.

Knoop, V L, S P Hoogendoorn, and J W C Van Lint. 2012. "Routing Strategies Based on Macroscopic Fundamental Diagram." Transportation Research Record: Journal of the Transportation Research Board 2315 (1): 1-10. http://dx.doi.org/10.3141/2315-01.

Knoop, Victor L, David De Jong, and Serge P Hoogendoorn. 2014. "Influence of Road Layout on Network Fundamental Diagram." Transportation Research Record: Journal of the Transportation Research Board 1 (2421): 22-30. http://dx.doi.org/10.3141/2421-03.

Knoop, Victor L, and Serge P Hoogendoorn. 2014. "Network Transmission Model: a dynamic traffic model at network level." In Transportation Research Board 93rd Annual Meeting, 14-1104, Washington DC.

Knoop, Victor L., Hans Van Lint, and Serge P. Hoogendoorn. 2015. "Traffic dynamics: Its impact on the Macroscopic Fundamental Diagram." Physica A: Statistical Mechanics and its Applications 438: 236-250. http://www.sciencedirect.com/science/article/pii/ S0378437115005695.

Lamotte, Raphael, and Nikolas Geroliminis. 2018. "The morning commute in urban areas with heterogeneous trip lengths." Transportation Research Part B: Methodological 117: 794-810. http://www.sciencedirect.com/science/article/pii/S0191261517307208.

Laval, Jorge A., and Ludovic Leclercq. 2013. "The Hamilton-Jacobi partial differential equation and the three representations of traffic flow." Transportation Research Part B: Methodological 52: 17-30. http://www.sciencedirect.com/science/article/pii/ S0191261513000349.

Leclercq, Ludovic, and Cecile Becarie. 2012. "Meso Lighthill-Whitham and Richards Model Designed for Network Applications." In Transportation Research Board 91st Annual Meet- 
ing, 12-0387, Washington DC. https://trid.trb.org/view/1128675.

Leclercq, Ludovic, Nicolas Chiabaut, and Beatrice Trinquier. 2014. "Macroscopic Fundamental Diagrams: A cross-comparison of estimation methods." Transportation Research Part B: Methodological 62: 1-12. http://www.sciencedirect.com/science/article/ $\mathrm{pii/S0191261514000174.}$

Leclercq, Ludovic, Celine Parzani, Victor L. Knoop, Jennifer Amourette, and Serge P. Hoogendoorn. 2015. "Macroscopic traffic dynamics with heterogeneous route patterns." Transportation Research Part C: Emerging Technologies 55: 292-307. http://www.sciencedirect. com/science/article/pii/S0968090X15001783.

Lentzakis, A. F., S. I. Ware, and R. Su. 2016. "Region-based dynamic forecast routing for autonomous vehicles." In 2016 IEEE 19th International Conference on Intelligent Transportation Systems (ITSC), Nov, 1464-1469.

Lighthill, M. J., and G. B. Whitham. 1955. "On Kinematic Waves II: A Theory of Traffic Flow on Long Crowded Roads." Proceedings of the Royal Society of London. Series A, Mathematical and Physical Sciences 229 (1178): 317-345.

Mahmassani, Hani, James C. Williams, and Robert Herman. 1984. "Investigation of network-level traffic flow relationships: Some simulation results." Transportation Research Record: Journal of the Transportation Research Board 971: 121-130. https://www.scopus.com/inward/record.uri?eid=2-s2.0-0021621747\&partnerID= 40\&md5=8165d659999e996ef878c1413162c149.

Mahmassani, H.S., T. Hou, and M. Saberi. 2013. "Connecting Networkwide Travel Time Reliability and the Network Fundamental Diagram of Traffic Flow." Transportation Research Record: Journal of the Transportation Research Board 2391: 80-91.

Mahut, Michael, Michael Florian, and Nicolas Tremblay. 2003. "Space-Time Queues and Dynamic Traffic Assignment: A model, algorithm and applications." In Transportation Research Board 82nd Annual Meeting, 03-1273, Washington DC.

Mazloumian, Amin, Nikolas Geroliminis, and Dirk Helbing. 2010. "The Spatial Variability of Vehicle Densities as Determinant of Urban Network Capacity." Philosophical Transactions of the Royal Society A: Mathematical, Physical and Engineering Sciences 368 (1928): 46274647.

Muhlich, Nicolas, Vikash V. Gayah, and Monica Menendez. 2015. "Use of Microsimulation for Examination of Macroscopic Fundamental Diagram Hysteresis Patterns for Hierarchical Urban Street Networks." Transportation Research Record: Journal of the Transportation Research Board 2491: 117-126.

Ortigosa, Javier, Monica Menendez, and Vikash V Gayah. 2015. "Analysis of Network Exit Functions for Various Urban Grid Network Configurations." Transportation Research Record: Journal of the Transportation Research Board 2491: 12-21.

Paipuri, Mahendra, Ludovic Leclercq, and Jean Krug. 2019. "Validation of Macroscopic Fundamental Diagrams-Based Models with Microscopic Simulations on Real Networks: Importance of Production Hysteresis and Trip Lengths Estimation." Transportation Research Record in press.

Richards, Paul L. 1956. "Shock Waves on the Highway." Operations Research 4 (1): 42-51.

Saberi, Meead, and Hani Mahmassani. 2012. "Exploring Properties of Networkwide FlowDensity Relations in a Freeway Network." Transportation Research Record: Journal of the Transportation Research Board 2315: 153-163.

Saeedmanesh, Mohammadreza, and Nikolas Geroliminis. 2016. "Clustering of heterogeneous networks with directional flows based on Snake similarities." Transportation Research Part B: Methodological 91: 250-269. http://www.sciencedirect.com/science/article/pii/ S0191261515302605.

Yildirimoglu, Mehmet, and Nikolas Geroliminis. 2014. "Approximating dynamic equilibrium conditions with macroscopic fundamental diagrams." Transportation Research Part B: Methodological 70: 186-200. http://www.sciencedirect.com/science/article/pii/ S0191261514001568.

Yildirimoglu, Mehmet, Mohsen Ramezani, and Nikolas Geroliminis. 2015. "Equilibrium anal- 
ysis and route guidance in large-scale networks with MFD dynamics." Transportation Research Part C: Emerging Technologies 59: 404-420. http://www.sciencedirect.com/ science/article/pii/S0968090X15001813.

Zhang, Lele, Timothy M Garoni, and Jan de Gier. 2013. "A comparative study of Macroscopic Fundamental Diagrams of arterial road networks governed by adaptive traffic signal systems." Transportation Research Part B: Methodological 49: 1-23. http://www. sciencedirect.com/science/article/pii/S0191261512001555.

Zhao, Tingting, Zhiheng Li, Bingyan Huang, Beipeng Mu, and Yi Zhang. 2014. "Exploring the influence of traveller information on macroscopic fundamental diagrams." Intelligent Transport Systems, IET 8 (1): 58-67. 